\title{
Analytical Benchmark Test Set for \\ Criticality Code Verification
}

\author{
Avneet Sood* \\ R. A. Forster \\ D. K. Parsons
}

${ }^{*}$ Graduate Research Assistant at Los Alamos. North Carolina State University,

Nuclear Engineering Department, Burlington Labs, Box 7909, Raleigh, NC 27695-7909.

\section{Los Alamos \\ NATIONAL LABORATORY}

Los Alamos, New Mexico 87545 



\section{DISCLAIMER}

This report was prepared as an account of work sponsored by an agency of the United States Government. Neither the United States Government nor any agency thereof, nor any of their employees, make any warranty, express or implied, or assumes any legal liability or responsibility for the accuracy, completeness, or usefulness of any information, apparatus, product, or process disclosed, or represents that its use would not infringe privately owned rights. Reference herein to any specific commercial product, process, or service by trade name, trademark, manufacturer, or otherwise does not necessarily constitute or imply its endorsement, recommendation, or favoring by the United States Government or any agency thereof. The views and opinions of authors expressed herein do not necessarily state or reflect those of the United States Government or any agency thereof. 


\section{DISCLAIMER}

Portions of this document may be illegible in electronic image products. Images are produced from the best available original document. 


\section{Contents}

I. INTRODUCTION 2

II. WHY THESE SOLUTIONS SERVE AS A TEST SET 2

III. SCOPE OF THE CRITICALITY VERIFICATION TEST SET 3

IV. USES OF THE CRITICALITY VERIFICATION TEST SET $\quad 8$

V. NEUTRON TRANSPORT EQUATION OVERVIEW 9

A. General $k_{e f f}$ Eigenvalue Equation . . . . . . . . . . . . . . . . . 9

B. One-Energy Group in One-Dimensional Slab Geometry . . . . . . . . . . . 10

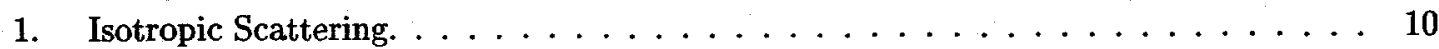

2. Linearly Anisotropic Scattering. . . . . . . . . . . . . . . . . 11

C. Two-Energy Groups in One-Dimensional Slab Geometry . . . . . . . . . . . . 12

1. Isotropic Scattering. . . . . . . . . . . . . . . . . 12

2. Linearly Anisotropic Scattering. . . . . . . . . . . . . . 13

VI. ONE-ENERGY GROUP PROBLEM DEFINITIONS AND RESULTS 15

A. One-Energy Group Isotropic Scattering . . . . . . . . . . . . . 16

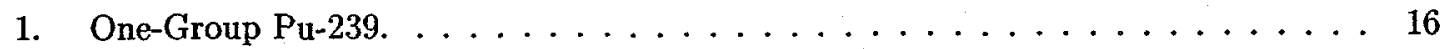

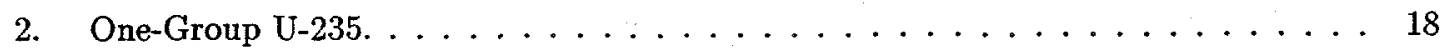

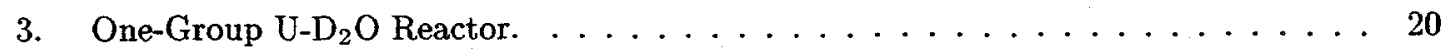

4. One-Group U-235 Reactor. . . . . . . . . . . . . . . . . 21

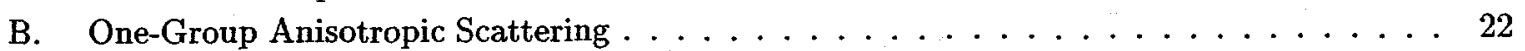

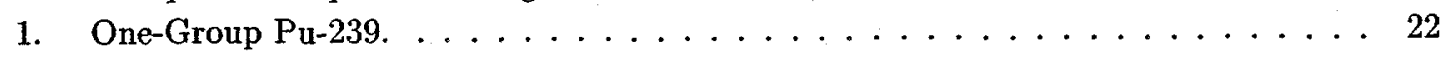

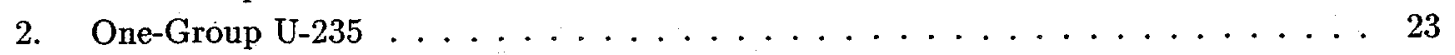

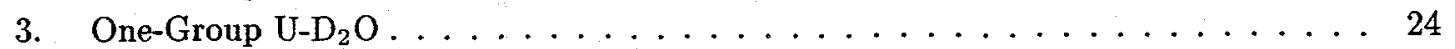

VII. TWO-ENERGY GROUP PROBLEM DEFINITIONS AND RESULTS 25

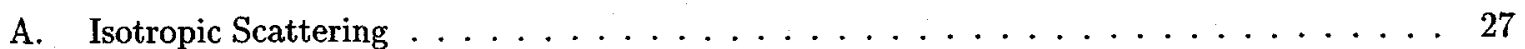

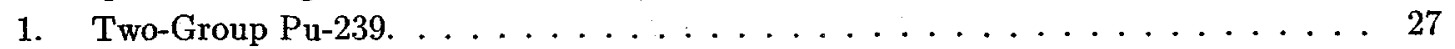

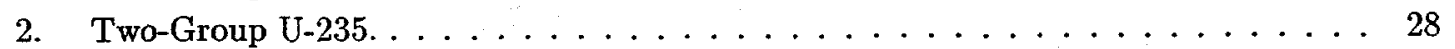

3. Two-Group Uranium-Aluminum-Water Assembly. . . . . . . . . . . . . . . 29

4. Two-Group Uranium Research Reactor. . . . . . . . . . . . . . . 30

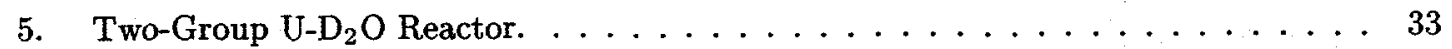

B. Linearly Anisotropic Scattering . . . . . . . . . . . . . . . . 34

1. Two-Group Uranium Research Reactor. . . . . . . . . . . . . . . . . . 34

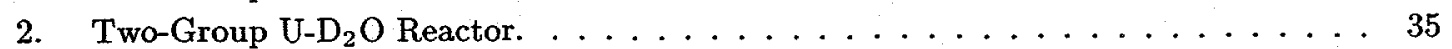

VIII. THREE-ENERGY GROUP PROBLEM RESULTS 36

IX. SIX-ENERGY GROUP PROBLEM DEFINITIONS AND RESULTS 37

$\begin{array}{ll}\text { X. SUMMARY } & 39\end{array}$

$\begin{array}{ll}\text { XI. ACKNOWLEDGMENTS } & 39\end{array}$

$\begin{array}{ll}\text { XII. REFERENCES } & \mathbf{4 0}\end{array}$ 
I. One-Energy Group Infinite Medium $k_{\infty} \ldots \ldots \ldots \ldots \ldots \ldots \ldots \ldots \ldots \ldots$

II. Two-Energy Group Infinite Medium $k_{\infty} \ldots \ldots \ldots \ldots \ldots \ldots \ldots$

III. Three-Energy Group Infinite Medium $k_{\infty}(\mathrm{a}) \ldots \ldots \ldots \ldots \ldots \ldots \ldots \ldots$

IV. Three-Energy Group Infinite Medium $k_{\infty}$ (b) $\ldots \ldots \ldots \ldots \ldots \ldots \ldots$

V. General Multigroup Infinite Medium $\mathbf{k}_{\infty}$ 


\section{List of Tables}

Nomenclature for Problem Identifiers . . . . . . . . . . . . . . . . . 4

Overview and Page Location for One-Energy Group Problem Identifiers . . . . . . . . . . . 6

Overview and Page Location for Two-Energy Group Problem Identifiers . . . . . . . . . . 7

Overview and Page Location for Three- and Six-Energy Group Problem Identifiers . . . . . 7 One-Group Macroscopic Cross Sections $\left(\mathrm{cm}^{-1}\right)$ for Pu-239 $(\mathrm{c}=1.40,1.50)$ and $\mathrm{H}_{2} \mathrm{O}(\mathrm{c}=0.90)$. 16 Critical Dimensions, $\mathrm{r}_{c}$, for One-Group Bare Pu-239 $(\mathrm{c}=1.50) \ldots \ldots \ldots \ldots \ldots$ Critical Dimensions, $\mathrm{r}_{c}$, for One-Group Bare Pu-239 $(\mathrm{c}=1.40) \ldots \ldots \ldots \ldots \ldots \ldots$ Normalized Scalar Fluxes for One-Group Bare Pu-239 $(\mathrm{c}=1.40) \ldots \ldots \ldots \ldots \ldots$ Critical Dimensions for One-Group Pu-239 Slab ( $c=1.50$ ) with Non-Symmetric $\mathrm{H}_{2} \mathrm{O}$ Reflector

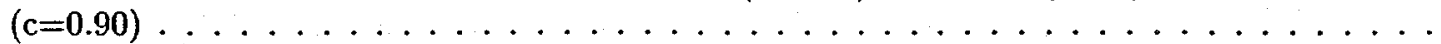
Critical Dimensions for One-Group Pu-239 Slab (c=1.50) with $\mathrm{H}_{2} \mathrm{O}$ Reflector $(\mathrm{c}=0.90) \ldots \ldots 17$ Critical Dimensions for One-Group Pu-239 Cylinder ( $c=1.40)$ with $\mathrm{H}_{2} \mathrm{O}$ Reflector $(c=0.90)$. 17 One-Group Macroscopic Cross Sections $\left(\mathrm{cm}^{-1}\right)$ for U-235 $(\mathrm{c}=1.30) \ldots \ldots \ldots \ldots$ Critical Dimensions, $r_{c}$, for One-Group Bare U-235 $(c=1.30) \ldots \ldots \ldots \ldots \ldots \ldots$ Normalized Scalar Fluxes for One-Group Bare U-235 $(\mathrm{c}=1.30) \ldots \ldots \ldots \ldots \ldots$ Critical Dimensions for One-Group U-235 Sphere with $\mathrm{H}_{2} \mathrm{O}$ Reflector $(\mathrm{c}=0.90) \ldots \ldots \ldots$ One-Group Macroscopic Cross Sections $\left(\mathrm{cm}^{-1}\right)$ for $\mathrm{U}-\mathrm{D}_{2} \mathrm{O}$ Reactor $(\mathrm{c}=1.02)$ and $\mathrm{H}_{2} \mathrm{O}(\mathrm{c}=0.90) 20$ Critical Dimensions, $\mathrm{r}_{c}$, for One-Group Bare U-D $\mathrm{D}_{2} \mathrm{O}$ Reactor $(\mathrm{c}=1.02) \ldots \ldots \ldots \ldots \ldots$ Normalized Scalar Fluxes for One-Group Bare U-D $\mathrm{D}_{2} \mathrm{O}$ Reactor $(\mathrm{c}=1.02) \ldots \ldots \ldots \ldots \ldots$ Critical Dimensions for One-Group U-D $\mathrm{D}_{2} \mathrm{O}(\mathrm{c}=1.02)$ Slab and Cylinder with $\mathrm{H}_{2} \mathrm{O}(\mathrm{c}=0.90)$ Reflector . . . . . . . . . . . . . . . . . . . . One-Group Macroscopic Cross Sections $\left(\mathrm{cm}^{-1}\right)$ for U-235 Reactor, Fe reflector, and $\mathrm{Na}$ Moderator . . . . . . . . . . . . . . . . . . . . . Critical Dimensions, $\mathbf{r}_{c}$, for One-Group U-235 Reactor $\ldots \ldots \ldots \ldots \ldots \ldots \ldots$ Critical Dimensions, $r_{c}$, for One-Group U-235 Reactor . . . . . . . . . . . . . . 21 Normalized Scalar Fluxes for One-Group U-235 Reactor . . . . . . . . . . . . . . . . 21 One-Group Macroscopic Anisotropic Cross Sections $\left(\mathrm{cm}^{-1}\right)$ for Pu-239 $(\mathrm{c}=1.40) \ldots \ldots 22$ Critical Dimensions, $\mathrm{r}_{c}$, for One-Group Bare Pu-239 $(\mathrm{c}=1.40) \ldots \ldots \ldots \ldots \ldots \ldots . \ldots . \ldots 22$ One-Group Macroscopic Anisotropic Cross Sections $\left(\mathrm{cm}^{-1}\right)$ for $\mathrm{U}-235(\mathrm{c}=1.30) \ldots \ldots$ Critical Dimensions, $\mathbf{r}_{c}$, for One-Group Bare U-235 $(\mathrm{c}=1.30) \ldots \ldots \ldots \ldots \ldots \ldots$ One-Group Macroscopic Anisotropic Cross Sections $\left(\mathrm{cm}^{-1}\right)$ for $\mathrm{U}-\mathrm{D}_{2} \mathrm{O}$ Reactor $\ldots \ldots \ldots .24$ Critical Dimensions, $\mathrm{r}_{c}$, for One-Group Bare $\mathrm{U}-\mathrm{D}_{2} \mathrm{O} \ldots \ldots \ldots \ldots \ldots \ldots \ldots$

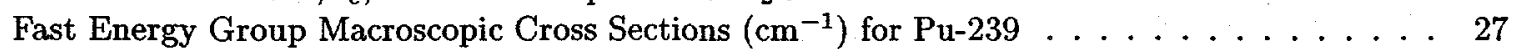
Slow Energy Group Macroscopic Cross Sections $\left(\mathrm{cm}^{-1}\right)$ for Pu-239 $\ldots \ldots \ldots \ldots \ldots$

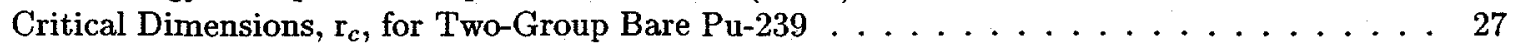
Fast Energy Group Macroscopic Cross Sections $\left(\mathrm{cm}^{-1}\right)$ for U-235 $\ldots \ldots \ldots \ldots \ldots$ Slow Energy Group Macroscopic Cross Sections $\left(\mathrm{cm}^{-1}\right)$ for U-235 $\ldots \ldots \ldots \ldots$

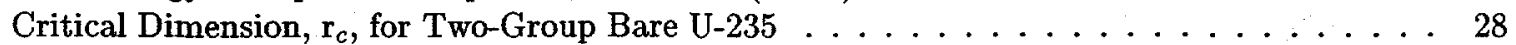

Fast Energy Group Macroscopic Cross Sections $\left(\mathrm{cm}^{-1}\right)$ for U-Al $\ldots \ldots \ldots \ldots \ldots$

Slow Energy Group Macroscopic Cross Sections $\left(\mathrm{cm}^{-1}\right)$ for U-Al $\ldots \ldots \ldots \ldots$

Critical Dimensions, $r_{c}$, for Two-Group Uranium-Aluminum-Water Assembly . . . . . . . 29

Fast Energy Group Macroscopic Cross Sections $\left(\mathrm{cm}^{-1}\right)$ for Research Reactor (a) . . . . . . 30 Slow Energy Group Macroscopic Cross Sections $\left(\mathrm{cm}^{-1}\right)$ for Research Reactor (a) . . . . . 30 Critical Dimensions, $r_{c}$, for Two-Group Bare Research Reactor (a) . . . . . . . . . . . 30 Normalized Scalar Fluxes for Two-Group Bare Research Reactor (a) . . . . . . . . . . . 30 Fast Energy Group Macroscopic Cross Sections $\left(\mathrm{cm}^{-1}\right)$ for Research Reactor (b), (c) and $\mathrm{H}_{2} \mathrm{O}$ Reflector (a) . . . . . . . . . . . . . . . . . . . . . 31 Slow Energy Group Macroscopic Cross Sections $\left(\mathrm{cm}^{-1}\right)$ for Research Reactor (b), (c) and

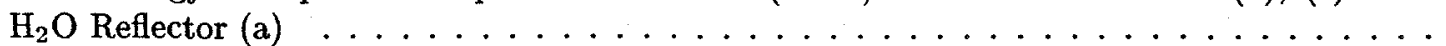
Critical Dimensions for Two-Group Research Reactor (b),(c) with $\mathrm{H}_{2} \mathrm{O}$ Reflector (a) . . . . Fast Group Macroscopic Cross Sections $\left(\mathrm{cm}^{-1}\right)$ for Research Reactor(d) and $\mathrm{H}_{2} \mathrm{O}$ Reflector (b), (c) . . . . . . . . . . . . . . . . . . . . . 32 
Slow Group Macroscopic Cross Sections $\left(\mathrm{cm}^{-1}\right)$ for Research Reactor(d) and $\mathrm{H}_{2} \mathrm{O}$ Reflector

(b), (c) . . . . . . . . . . . . . . . . . . . . . . 32

48 Critical Dimensions for Two-Group Infinite Slab Lattice Cell and $\mathrm{H}_{2} \mathrm{O}$ Reflector (b), (c) . . . 32

49 Fast Energy Group Macroscopic Cross Sections $\left(\mathrm{cm}^{-1}\right)$ for $\mathrm{U}-\mathrm{D}_{2} \mathrm{O} \ldots \ldots \ldots \ldots$

50 Slow Energy Group Macroscopic Cross Sections $\left(\mathrm{cm}^{-1}\right)$ for $U-D_{2} \mathrm{O} \ldots \ldots \ldots \ldots$

51 Critical Dimension, $\mathbf{r}_{c}$, for Two-Group $\mathrm{D}_{2} \mathrm{O}$ System $\ldots \ldots \ldots \ldots \ldots \ldots \ldots$

52 Fast Group Cross Sections for Linearly Anisotropic Scattering $\left(\mathrm{cm}^{-1}\right)$ Research Reactor (a) . 34

53 Slow Group Cross Sections for Linearly Anisotropic Scattering $\left(\mathrm{cm}^{-1}\right)$ Research Reactor (a) . 34

54 Critical Dimension, $r_{c}$, for Two-Group Linearly Anisotropic Scattering Research Reactor (a) . 34

55 Normalized Scalar Fluxes for Two-Group Bare Research Reactor (a) . . . . . . . . . . . . 34

56 Fast Energy Group Cross Sections for Linearly Anisotropic Scattering $\left(\mathrm{cm}^{-1}\right)$ for $\mathrm{U}_{-} \mathrm{D}_{2} \mathrm{O} \quad \ldots \quad 35$

57 Slow Energy Group Cross Sections for Linearly Anisotropic Scattering $\left(\mathrm{cm}^{-1}\right)$ for U- $\mathrm{D}_{2} \mathrm{O}$. . 35

58 Critical Dimension, $\mathrm{r}_{c}$, for Two-Group Linearly Anisotropic Scattering for $\mathrm{U}_{-} \mathrm{D}_{2} \mathrm{O}$ Reactor . 35

59 Fast Energy Group Macroscopic Cross Sections $\left(\mathrm{cm}^{-1}\right)$ for Research Reactor . . . . . . . 36

60 Middle Energy Group Macroscopic Cross Sections $\left(\mathrm{cm}^{-1}\right)$ for Research Reactor . . . . . . . 36

61 Slow Energy Group Macroscopic Cross Sections $\left(\mathrm{cm}^{-1}\right)$ for Research Reactor . . . . . . . 36

62 Fast Energy Group 6 Macroscopic Cross Sections $\left(\mathrm{cm}^{-1}\right)$ for Research Reactor . . . . . . 37

63 Energy Group 5 Macroscopic Cross Sections $\left(\mathrm{cm}^{-1}\right)$ for Research Reactor . . . . . . . . . 37

64 Energy Group 4 Macroscopic Cross Sections $\left(\mathrm{cm}^{-1}\right)$ for Research Reactor . . . . . . . . . 37

65 Energy Group 3 Macroscopic Cross Sections $\left(\mathrm{cm}^{-1}\right)$ for Research Reactor . . . . . . . . . 37

66 Energy Group 2 Macroscopic Cross Sections $\left(\mathrm{cm}^{-1}\right)$ for Research Reactor . . . . . . . . . 37

67 Slow Energy Group 1 Macroscopic Cross Sections $\left(\mathrm{cm}^{-1}\right)$ for Research Reactor . . . . . . 37

\section{List of Figures}

1 Critical Dimension, $\mathbf{r}_{c}$, for Bare One-Dimensional Geometries and Infinite Slab Lattice Cell . 


\section{ANALYTICAL BENCHMARK TEST SET FOR CRITICALITY CODE VERIFICATION}

by

A. Sood (NCSU), R.A. Forster (LANL), and D.K. Parsons (LANL)

\section{ABSTRACT}

A number of published numerical solutions to analytic eigenvalue $\left(\mathbf{k}_{\text {eff }}\right)$ and eigenfunction equations are summarized for the purpose of creating a criticality verification benchmark test set. The 75-problem test set allows the user to verify the correctness of a criticality code for infinite medium and simple geometries in one- and two-energy groups, one- and two-media, and both isotropic and anisotropic neutron scattering. The problem specifications will produce both $k_{e f f}=1$ and the quoted $k_{\infty}$ to at least five decimal places. Additional uses of the test set for code verification are also discussed. A list of 45 references and an appendix with $\mathbf{k}_{\infty}$ derivations is also included. 


\section{INTRODUCTION}

This report describes a set of benchmark problems with analytic eigenvalue $\left(k_{e f f}\right)$ and eigenfunction (flux) solutions to the neutron transport equation from peer-reviewed journal articles. The purpose of the test set is to verify that transport algorithms and codes can correctly calculate the analytic $k_{e f f}$ and fluxes to at least five decimal places. These test set problems for infinite medium, slab, cylindrical, and spherical geometries in one- and two-energy groups, one- and two-media, and both isotropic and linearly anisotropic scattering are completely described using the listed references in this report. A three-group infinite medium and a six-group variant $k_{\infty}$ problem (unpublished) are also included.

Verification is defined as "the process of evaluating a system or component to determine whether the products of a given development phase satisfy the conditions imposed at the start of the phase" ${ }^{1}$ or as a "proof of correctness." Confirmation (proof) of correctness is "a formal technique used to prove mathematically that a computer program satisfies its specified requirements." ${ }^{1}$ In contrast to verification, validation is defined as "the process of evaluating a system or component during or at the end of the development process to determine whether it satisfies specified requirements." ${ }^{1}$ Thus code verification checks that the intended calculations have been executed correctly, while code validation compares the calculated results with experimental data.

The objectives of this report are to define and document a set of analytic benchmarks for verifying criticality codes. Benchmark is defined as "a standard against which measurement or comparisons can be made." ${ }^{1}$ Available benchmarks for code verification do not focus on criticality problems. ${ }^{2}$ Validation benchmarks from critical experiments do exist, but are not verification benchmarks. ${ }^{3}$ Initial efforts to compile a benchmark test set for criticality calculation verification was begun, but not completed. ${ }^{4,5}$ The analytic benchmarks described here can be used to verify computed numerical solutions for $k_{e f f}$ and the associated flux with virtually no uncertainty in the numerical benchmark values.

\section{WHY THESE SOLUTIONS SERVE AS A TEST SET}

All critical dimensions, $\mathrm{k}_{\text {eff }}$, and scalar neutron flux results quoted here are based on numerical computations using the analytic solutions to the $\mathrm{k}_{\text {eff }}$ eigenvalue (homogeneous) transport equation for "simple" problems. The analytic methods used include Case's singular eigenfunction, ${ }^{6} \mathrm{~F}_{N}$ and $\mathrm{B}_{N}$ methods, ${ }^{7,8}$ and Green's functions. ${ }^{9}$ All of these test set problem specifications and results are from peer-reviewed journals, and have, in some cases, been solved numerically using more than one analytic solution. All calculated values for critical dimensions, $\mathrm{k}_{e f f}$, and the scalar neutron flux are believed to be accurate to at least five decimal places. 


\section{SCOPE OF THE CRITICALITY VERIFICATION TEST SET}

The verification test set was chosen to represent a "wide" range of problems from the relatively small number of published solutions. These problems include simple geometries, few neutron energy groups, and simplified (isotropic and linearly anisotropic) scattering models. The problems use neutron cross sections that are reasonable representations of the materials described. These cross sections are not general purpose multi-group values. The cross sections are used because they are extracted from the literature results and are intended to be used only to verify algorithm performance and not to predict criticality experiments.

The basic geometries include an infinite medium, slab, cylinder, and sphere with one- and two-energy group representations of uniform homogeneous materials. The slab and cylinder geometries are one-dimensional, as shown in Fig. 1; that is, each is finite in one dimension (thickness for slab and radius for the cylinders) and infinite elsewhere. The two-media problems surround each geometry with a specified thickness of reflector. Solutions for one-, two-, and three-group infinite medium problems are derived in Appendix A.

The critical dimension, $\mathbf{r}_{c}$, is defined pictorially for the one-dimensional, one-medium problem geometries in Fig. 1, as well as the two-media infinite slab lattice cell. Reflector dimension(s) are provided for the reflected cases.

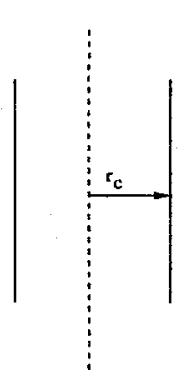

Infinite Slab

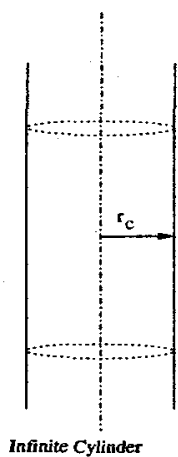

Infinite Cylinder

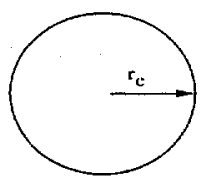

Sphere

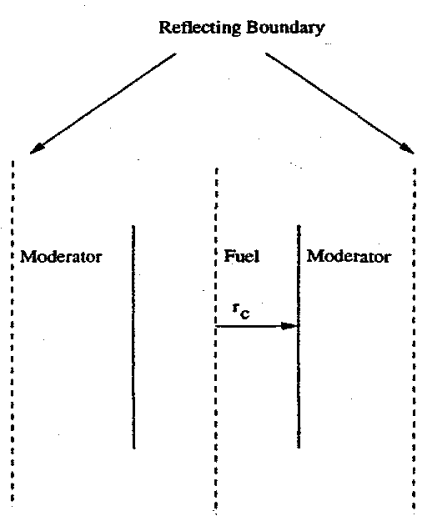

Infinite Slab Latice Cell

Figure 1: Critical Dimension, $\mathrm{r}_{c}$, for Bare One-Dimensional Geometries and Infinite Slab Lattice Cell

The emphasis of the test set is on the fundamental eigenvalue, $\mathrm{k}_{\text {eff }}$. All $\mathrm{k}_{\text {eff }}$ eigenvalues for finite fissile materials are unity to at least five decimal places. The $\mathbf{k}_{\infty}$ values for a uniform homogeneous infinite medium are greater than unity. Few numerical eigenfunction solutions are published; consequently, mainly one-group and uniform homogeneous infinite medium fluxes are included in the test set results.

To assist in verification, each problem has a unique problem number and identifier. Since the test set includes bare and multi-media problems, there are two forms of the identifier. The first form is for a bare geometry: 


\section{Fissile Material - Energy Groups - Scattering - Geometry}

The possible entries for each category are listed in Table 1. The fissile materials and identifier consist of $\mathrm{Pu}-$ 239 (PU), U-235 (U), highly enriched uranium-aluminum-water assembly (UAL), low enrichment uranium and $\mathrm{D}_{2} \mathrm{O}$ reactor system (UD2O), and a highly enriched uranium research reactor (URR). The identifier may be followed by a letter to differentiate between different cross-section sets from nominally the same material. The table lists identifiers for the reflector material (if any), number of energy groups, scattering order, and geometry. The geometry is identified by the first two letters in the table. The exception is for the infinite slab lattice cell which uses ISLC. An example of the one material form of the identifier is:

\section{U-2-0-SP}

which is the identifier for a bare U-235 reactor (no reflector), 2 energy groups, isotropically scattering, in spherical geometry.

The second form of the identifier includes the reflecting material. The reflectors are usually $\mathrm{H}_{2} \mathrm{O}$ with an exception of a three region $\mathrm{Fe}, \mathrm{Na}$, $\mathrm{Fe}$ reflector. Although many of the reflectors are identified as $\mathrm{H}_{2} \mathrm{O}$, the reflector cross sections are often unique to each problem. Consequently, a letter may follow $\mathrm{H} 2 \mathrm{O}$ indicating the $\mathrm{H}_{2} \mathrm{O}$ cross-section set used. The multi-media identifier form is:

\section{Fissile Material - Reflecting Material (thickness) - Energy Groups - Scattering - Geometry}

To separate multiple reflector thicknesses for the same fissile material, the thickness is given in parenthesis in the title in units of mean free paths (mfp). For example,

\section{UD2O-H2O(10)-1-0-SL}

is the identifier for a uranium and $\mathrm{D}_{2} \mathrm{O}$ reactor with a $\mathrm{H}_{2} \mathrm{O}$ reflector of 10 mean free path thickness, oneenergy group, isotropically scattering, in slab geometry. An "IN" in parenthesis after the $\mathrm{H} 2 \mathrm{O}$ means an infinite water reflector.

Table 1: Nomenclature for Problem Identifiers

\begin{tabular}{|c|c|l|l|l|}
\hline Fissile Material & Reflector Material & Energy Groups & Scattering Order & Geometry \\
\hline PU & bare & 1 group & $0-\mathrm{P}_{0}$ Isotropic & INfinite \\
U & H2O & 2 groups & $1-\mathrm{P}_{1}$ Anisotropic & SLab \\
UD2O & Fe-Na & 3 groups & $2-\mathrm{P}_{2}$ Anisotropic & CYYlinder \\
UAL & & 6 groups & & $\underline{\text { SPhere }}$ \\
URR & & & & Infinite Slab Lattice Cell \\
\hline
\end{tabular}

Tables 2,3 , and 4 summarize each of the 75 problems in the test set and give the page number in this report. An " $x$ " in the "Flux" column appears if the associated normalized spatial neutron fluxes or energy group flux ratio are given. An asterisk in the "Scattering" column appears if the $P_{1}$ scattering expansion 
becomes negative over part of the $\mu$ interval from -1 to 1 . The purpose of the layout of the test set problems is to increase user-friendliness. The user has all pertinent information to define any given problem in the verification test set on a single page.

There are 43 problems in the one-energy group case: 30 problems assume isotropic scattering and 13 have anisotropic scattering. For the two-energy group problems, there are 30 problems subdivided into 26 isotropic scattering problems and 4 linearly anisotropic problems. Also included for an infinite medium are a three-group and a six-group ( 2 coupled sets of three groups) isotropic problem. The test set includes 24 infinite medium problems, 24 slabs, 9 one-energy group cylinders, 14 spheres, and 4 infinite slab lattice cells. 
Table 2: Overview and Page Location for One-Energy Group Problem Identifiers

\begin{tabular}{|c|l|c|c|c|}
\hline Number & Problem Identifier & Flux & Page Number & Scattering \\
\hline 1 & PUa-1-0-IN & $\mathrm{x}$ & 16 & \\
2 & PUa-1-0-SL & & 16 & \\
3 & PUa-H2O(1)-1-0-SL & & 17 & \\
4 & PUa-H2O(0.5)-1-0-SL & & 17 & \\
5 & PUb-1-0-IN & $\mathrm{x}$ & 16 & \\
6 & PUb-1-0-SL & $\mathrm{x}$ & 16 & \\
7 & PUb-1-0-CY & $\mathrm{x}$ & 16 & \\
8 & PUb-1-0-SP & $\mathrm{x}$ & 16 & \\
9 & PUb-H2O(1)-1-0-CY & & 17 & \\
10 & PUb-H2O(10)-1-0-CY & & 17 & \\
11 & Ua-1-0-IN & $\mathrm{x}$ & 18 & \\
12 & Ua-1-0-SL & $\mathrm{x}$ & 18 & \\
13 & Ua-1-0-CY & & 18 & \\
14 & Ua-1-0-SP & $\mathrm{x}$ & 18 & \\
15 & Ub-1-0-IN & $\mathrm{x}$ & 18 & \\
16 & Ub-H2O(1)-1-0-SP & & 19 & \\
17 & Uc-1-0-IN & $\mathrm{x}$ & 18 & \\
18 & Uc-H2O(2)-1-0-SP & & 19 & \\
19 & Ud-1-0-IN & $\mathrm{x}$ & 18 & \\
20 & Ud-H2O(3)-1-0-SP & & 19 & \\
21 & UD2O-1-0-IN & $\mathrm{x}$ & 20 & \\
22 & UD2O-1-0-SL & $\mathrm{x}$ & 20 & \\
23 & UD2O-1-0-CY & & 20 & \\
24 & UD2O-1-0-SP & $\mathrm{x}$ & 20 & \\
25 & UD2O-H2O(1)-1-0-SL & & 20 & \\
26 & UD2O-H2O(10)-1-0-SL & & 20 & \\
27 & UD2O-H2O(1)-1-0-CY & & 20 & \\
28 & UD2O-H2O(10)-1-0-CY & & 20 & \\
29 & Ue-1-0-IN & $\mathrm{x}$ & 21 & \\
30 & Ue-Fe-Na-1-0-SL & $\mathrm{x}$ & 21 & \\
31 & PU-1-1-IN & $\mathrm{x}$ & 22 & \\
32 & PUa-1-1-SL & & 22 & \\
33 & PUa-1-2-SL & & 22 & \\
34 & PUb-1-1-SL & & 22 & \\
35 & PUb-1-2-SL & & 22 & \\
36 & Ua-1-1-CY & & \\
37 & Ub-1-1-CY & & \\
38 & UD2Oa-1-1-IN & & 23 & \\
39 & UD2Oa-1-1-SP & & 24 & \\
40 & UD2Ob-1-1-IN & & 24 & \\
41 & UD2Ob-1-1-SP & & 24 & \\
42 & UD2Oc-1-1-IN & & 24 & \\
43 & UD2Oc-1-1-SP & & 24 & \\
\hline
\end{tabular}

${ }^{*} \mathrm{P}_{1}$ scattering expansion becomes negative over part of the $\mu$ interval from -1 to 1 . 
Table 3: Overview and Page Location for Two-Energy Group Problem Identifiers

\begin{tabular}{|c|l|c|c|c|}
\hline Number & Problem Identifier & Flux & Page Number & Scattering \\
\hline 44 & PU-2-0-IN & $\mathrm{x}$ & 27 & \\
45 & PU-2-0-SL & & 27 & \\
46 & PU-2-0-SP & $\mathrm{x}$ & 27 & \\
47 & U-2-0-IN & & 28 & \\
48 & U-2-0-SL & & 28 & \\
49 & U-2-0-SP & $\mathrm{x}$ & 29 & \\
50 & UAL-2-0-IN & & 29 & \\
51 & UAL-2-0-SL & 29 & \\
52 & UAL-2-0-SP & $\mathrm{x}$ & 30 & \\
53 & URRa-2-0-IN & $\mathrm{x}$ & 30 & \\
54 & URRa-2-0-SL & & 30 & \\
55 & URRa-2-0-SP & $\mathrm{x}$ & 31 & \\
56 & URRb-2-0-IN & & 31 & \\
57 & URRc-2-0-IN & 31 & Isotropic \\
58 & URRb-H2Oa(1)-2-0-SL & & 31 & \\
59 & URRb-H2Oa(5)-2-0-SL & & 31 & \\
60 & URRb-H2Oa(IN)-2-0-SL & & 31 & \\
61 & URRc-H2Oa(IN)-2-0-SL & & 32 & \\
62 & URRd-2-0-IN & $\mathrm{x}$ & 32 & \\
63 & URRd-H2Ob(1)-2-0-ISLC & & 32 & \\
64 & URRd-H2Ob(10)-2-0-ISLC & & 32 & \\
65 & URRd-H2Oc(1)-2-0-ISLC & & 32 & \\
66 & URRd-H2Oc(10)-2-0-ISLC & & 33 & \\
67 & UD2O-2-0-IN & $\mathrm{x}$ & 33 & \\
68 & UD2O-2-0-SL & & 33 & \\
69 & UD2O-2-0-SP & $\mathrm{x}$ & 34 & \\
70 & URRa-2-1-IN & & \\
71 & URRa-2-1-SL & & \\
72 & UD2O-2-1-IN & & \\
73 & UD2O-2-1-SL & & \\
\end{tabular}

${ }^{*} P_{1}$ scattering expansion becomes negative over part of the $\mu$ interval from -1 to 1 .

Table 4: Overview and Page Location for Three- and Six-Energy Group Problem Identifiers

\begin{tabular}{|c|l|c|c|c|}
\hline Number & Problem Identifier & Flux & Page Number & Scattering \\
\hline 74 & URR-3-0-IN & $\mathrm{x}$ & 36 & Isotropic \\
75 & URR-6-0-IN & $\mathrm{x}$ & 38 & \\
\hline
\end{tabular}




\section{USES OF THE CRITICALITY VERIFICATION TEST SET}

This report provides all necessary problem definitions and published critical $\left(\mathrm{k}_{\text {eff }}=1\right)$ dimensions, $\mathrm{k}_{\infty}$, and scalar neutron flux results to verify a criticality transport algorithm or code and associated numerics such as random number generation and round-off errors. All material cross sections provided are macroscopic, so the atom density used by the code should be unity. Not all of the analytic solutions from the references are used, however, because the number of problems in the test set becomes too large. For other solutions not included in this report, see the reference list.

The verification test set problems can be used in several ways. The user can choose to simply calculate the problems and compare forward and adjoint $\mathrm{k}_{\text {eff }}$ and neutron flux results with the benchmark solutions. However, there are several more verification processes that could be included. For example, in Monte Carlo codes, both multi-group and pointwise representation of multi-group data can be used. In multi-group problems, an alternative verification procedure is to change the energy group structure when up-scattering is allowed; that is, reverse the order of the fast and slow groups. To examine the alpha eigenvalue or timedependent neutron decay or growth, the capture and total cross sections can be modified by $\alpha / v$ to represent subcritical and supercritical systems.

Another part of code verification is testing different representations of the same geometry (e.g., reflecting boundaries and lattices). An example is an infinite one-dimensional slab (finite in one dimension and infinite in the other two dimensions) as shown in Fig. 1, which could be modeled as a three-dimensional cube with four reflective boundaries. Other geometry options can be tested by constructing several smaller cubes inside of the three-dimensional representation of a one-dimensional critical slab. The infinite medium problem can be represented by using large geometric boundaries, reflecting boundaries, or infinite lattices of finite shapes. Infinite medium problems can be used to verify constant scalar and angular flux in each energy group as well as scalar flux ratios for more than one energy group. Three-dimensional geometric representations of optically small objects can also be tested for $k_{\infty}$ in infinite medium problems. ${ }^{10}$ Purely absorbing one-group infinite medium problems can provide faster code verification since scattering does not alter the infinite medium $k_{\infty}$ (see Appendix A).

Another use of this verification set includes testing of any flux tally approximations. This can be especially important at near tangential angles where some codes assume an average value for the cosine of the angle. This can also affect $k_{e f f}$ if it is estimated by same section of code that calculates the flux.

Different calculation capabilities of a code should be tested using these problems. For Monte Carlo codes, different variance reduction methods such as analog or implicit capture and geometric splitting or Russian roulette can be verified. Cycle-to-cycle correlations in the estimated $\mathbf{k}_{\text {eff }}$ standard deviation must be taken into account to form valid $\mathrm{k}_{\text {eff }}$ confidence intervals. Statistically independent runs can be made and analyzed if necessary. The magnitude of any negative bias in $k_{e f f}$, which is a function of the number of neutron histories per fission generation, also needs to be considered and made smaller than $0.00001 .{ }^{11}$ 
Deterministic codes can assess convergence characteristics and correctness of $k_{e f f}$ and the flux as a function of space and angle representation. Various characteristics of discrete ordinates numerics can also be checked such as the effects of eigenvalue search algorithms, angular redistribution terms in curvilinear geometries, ray effects, and various alternative geometric descriptions.

\section{NEUTRON TRANSPORT EQUATION OVERVIEW}

The neutron transport equation being solved in these benchmark problems is briefly described for oneand two-energy groups and the isotropic and linearly anisotropic cases. The infinite medium solutions for $\mathrm{k}_{\infty}$ and the flux ratios are described in Appendix A.

\section{A. General $\mathbf{k}_{\text {eff }}$ Eigenvalue Equation}

The steady state neutron transport equation can be written as a $\mathrm{k}_{\text {eff }}$ eigenvalue problem as: ${ }^{12}$

$$
\begin{aligned}
\vec{\Omega} \cdot \nabla \Psi(\vec{r}, E, \vec{\Omega})+\Sigma_{t}(\vec{r}, E) \Psi(\vec{r}, E, \vec{\Omega})= & \int_{0}^{\infty} d E^{\prime} \int_{4 \pi} d \vec{\Omega}^{\prime} \Sigma_{s}\left(\vec{r}, E^{\prime} \rightarrow E, \vec{\Omega}^{\prime} \rightarrow \vec{\Omega}\right) \Psi\left(\vec{r}, E^{\prime}, \vec{\Omega}^{\prime}\right) \\
& +\chi(E) \int_{0}^{\infty} d E^{\prime} \frac{\nu\left(\vec{r}, E^{\prime}\right)}{4 \pi \mathrm{k}_{\mathrm{eff}}} \Sigma_{f}\left(\vec{r}, E^{\prime}\right) \int_{4 \pi} \Psi\left(\vec{r}, E^{\prime}, \vec{\Omega}^{\prime}\right) d \vec{\Omega}^{\prime}
\end{aligned}
$$

where:

$$
\begin{aligned}
\Psi(\vec{r}, E, \vec{\Omega})= & \text { angular neutron flux as a function of space } \vec{r}, \text { energy } \mathrm{E}, \text { and angle } \vec{\Omega} \\
\Sigma_{t}(\vec{r}, E)= & \text { total neutron macroscopic cross section } \\
\Sigma_{s}\left(\vec{r}, E^{\prime} \rightarrow E, \vec{\Omega}^{\prime} \rightarrow \vec{\Omega}\right) d E d \vec{\Omega}= & \text { neutron scattering macroscopic cross section } \\
& \text { from } \mathrm{E}^{\prime} \text { to } \mathrm{E}+\mathrm{dE} \text { in direction } \mathrm{d} \vec{\Omega}^{\prime} \text { about } \vec{\Omega} \\
= & \Sigma_{\text {elastic }}+\Sigma_{\left(n, n^{\prime}\right)}+2 \Sigma_{(n, 2 n)}+\ldots \\
\Sigma_{f}\left(\vec{r}, E^{\prime}\right)= & \text { neutron fission macroscopic cross section } \\
\nu\left(\vec{r}, E^{\prime}\right)= & \text { number of neutrons emitted from each fission event } \\
\chi(E)= & \text { fission neutron energy distribution }
\end{aligned}
$$

For these test problems, there are no $\left(n, x n^{\prime}\right)$ reactions, $x>1$, included in $\Sigma_{s}$. The scattering cross section includes only isotropic scattering and no higher order scattering components. Therefore, $\Sigma_{c}=\Sigma_{t}-\Sigma_{s}-\Sigma_{f}$, where $\Sigma_{c}$ is the neutron capture cross section (zero neutrons emitted). This report also provides values for the scalar neutron flux, which is defined as $\phi(r, E)=\int_{\Omega} \Psi(\vec{r}, E, \vec{\Omega}) d \vec{\Omega}$. The reported scalar neutron flux values are normalized to the flux at the center of the fissile material.

The correct solution for $\mathbf{k}_{\text {eff }}$ will provide equality or balance for Eq. 1: losses equal gains. The $\mathbf{k}_{\text {eff }}$ eigenvalue is only associated with the fission reaction and no other multiplying process such as $(n, 2 n)$. The 
fundamental eigenvalue, $\mathbf{k}_{e f f}$, is unity for a critical system, less than unity for a subcritical system, and greater than unity for a supercritical system. The steady state $\mathrm{k}_{\text {eff }}$ eigenvalue equation is physically correct only when $\mathrm{k}_{e f f}$ is unity and there is no decay or growth in $\Psi(r, E, \vec{\Omega})$. A solution when $\mathrm{k}_{\text {eff }}$ is not unity is still a valuable indicator of the ability of a system to sustain a fission chain reaction. When an infinite medium is considered, $k_{e f f}$ will be referred to as $k_{\infty}$. This report gives results for the fundamental $k_{e f f}$ eigenvalue. For higher eigenvalue results, see Refs. 13,14,15,17,18,19,20.

Analytic solutions to this general form of the transport equation do not exist. If we begin to simplify the equation by considering a one-dimensional slab with azimuthally symmetric scattering, the transport equation reduces to:

$$
\begin{aligned}
\mu \frac{\partial \Psi(x, E, \mu)}{\partial x}+\Sigma_{t}(x, E) \Psi(x, E, \mu)= & 2 \pi \int_{0}^{\infty} d E^{\prime} \int_{-1}^{1} \Sigma_{s}\left(x, E^{\prime} \rightarrow E, \mu, \mu^{\prime}\right) \Psi\left(x, E^{\prime}, \mu^{\prime}\right) d \mu^{\prime} \\
& +\chi(E) \int_{0}^{\infty} d E^{\prime} \frac{\nu\left(x, E^{\prime}\right)}{2 \mathrm{k}_{\mathrm{eff}}} \Sigma_{f}\left(x, E^{\prime}\right) \int_{-1}^{1} \Psi\left(r, E^{\prime}, \mu^{\prime}\right) d \mu^{\prime}
\end{aligned}
$$

where $\mu$ is the cosine of the angle relative to a given direction.

For the other finite geometries, the slab leakage term, $\mu \frac{\partial \Psi}{\partial x}$ must be replaced with the appropriate leakage term for a cylinder or sphere. ${ }^{12}$ This equation is still too difficult to solve analytically. Approximations are required in both the space, angle, and energy variables to allow an analytic solution.

\section{B. One-Energy Group in One-Dimensional Slab Geometry}

\section{Isotropic Scattering.}

To simplify Eq. 2, isotropic scattering is assumed, which implies that the emission angle after scattering is equally likely in all directions, giving:

$$
\Sigma_{s}\left(\vec{r}, E^{\prime} \rightarrow E, \vec{\Omega}^{\prime} \rightarrow \vec{\Omega}\right)=\frac{1}{4 \pi} \Sigma_{s}\left(r, E^{\prime} \rightarrow E\right)
$$

The transport equation can be further simplified by assuming a homogeneous medium and only one energy group. Using these additional assumptions to define one-group scalar flux weighted average cross sections, the transport equation becomes: ${ }^{12}$

$$
\mu \frac{\partial \Psi(x, \mu)}{\partial x}+\Sigma_{t} \Psi(x, \mu)=\frac{\Sigma_{s}}{2} \int_{-1}^{1} \Psi\left(x, \mu^{\prime}\right) d \mu^{\prime}+\frac{\nu \Sigma_{f}}{2 \mathrm{k}_{\mathrm{eff}}} \int_{-1}^{1} \Psi\left(x, \mu^{\prime}\right) d \mu^{\prime}
$$

A convenient transformation for this form of the transport equation is to change the position variable into a mean free path, or optical thickness, z. This change is done by using $z=\Sigma_{t} x$, thereby making $\partial z / \partial x=\Sigma_{t}$. 
If we divide the transport equation by $\Sigma_{t}$, and use the change of variables, the neutron transport equation becomes:

$$
\frac{\mu}{\Sigma_{t}} \frac{\partial \Psi(z, \mu)}{\partial z}\left(\frac{\partial z}{\partial x}\right)+\Psi(z, \mu)=\frac{\Sigma_{s}+\frac{\nu \Sigma_{f}}{k_{\text {eff }}}}{2 \Sigma_{t}} \int_{-1}^{1} \Psi\left(z, \mu^{\prime}\right) d \mu^{\prime}
$$

Notice that the dimensionless position variable, $z$, is now defined in terms of mean free paths (mfp) (i.e., $1 / \Sigma_{t}$ ). The final simplified one-energy group transport equation for a slab can be written in a slightly more convenient form as:

$$
\mu \frac{\partial \Psi(z, \mu)}{\partial z}+\Psi(z, \mu)=\frac{c}{2} \int_{-1}^{1} \Psi\left(z, \mu^{\prime}\right) d \mu^{\prime}
$$

where:

$$
c=\frac{\Sigma_{s}+\frac{\nu \Sigma_{f}}{k_{\text {eff }}}}{\Sigma_{t}}
$$

The parameter $c$ is defined as the mean number of secondary neutrons produced per neutron reaction and is also known as the secondaries ratio. This equation is the form of the one-medium, one-energy group transport equation for a slab. This equation still requires elaborate mathematics to solve as reported in the literature. Derivation of the one-energy group $\mathrm{k}_{\infty}$ solution for the infinite medium case is shown in Appendix A. A $c$ of unity is equivalent to a $k_{\infty}$ of unity. Thus, $c$ must be greater than unity to have a finite critical system.

The literature uses this form of the neutron transport equation with a non-reentrant boundary condition to derive one-energy group, isotropic scattering analytic solutions for the critical $\left(k_{e f f}=1\right)$ dimensional scalar neutron flux. It should be noted that $c$ values for $k_{\text {eff }}=1$ in Eq. 6 are presented in the literature, thereby making $c=\frac{\Sigma_{s}+\nu \Sigma_{f}}{\Sigma_{\varepsilon}}$. The typical range of $c$ found in the literature for fissile materials is from 1.01 to 2.00. The one-group cross sections selected for the test set mimic the physical characteristics of the two-group problems and range from 1.02 to 1.50. A value of $c$ of 1.5 is the upper limit for real fissile materials.

\section{Linearly Anisotropic Scattering.}

The scattering term, $\Sigma_{s}\left(\vec{r}, E^{\prime} \rightarrow E, \vec{\Omega}^{\prime} \rightarrow \vec{\Omega}\right)$ can be a strong function of the cosine of the scattering angle, $\mu_{0}=\vec{\Omega}^{\prime} \cdot \vec{\Omega}$. The angular dependence can be analyzed by Legendre polynomial series expansion of $\Sigma_{s}\left(\vec{r}, E^{\prime} \rightarrow E, \vec{\Omega}^{\prime} \cdot \vec{\Omega}\right) .{ }^{21}$ Using the Legendre polynomial expansion, the one-energy group, one-dimensional slab, neutron transport equation can be written in a similar form to Eq. $5:^{22,23}$

$$
\mu \frac{\partial \Psi(z, \mu)}{\partial z}+\Psi(z, \mu)=\frac{c}{2} \int_{-1}^{1} \Psi\left(z, \mu^{\prime}\right)\left(1+\mu^{\prime} \mu\right) d \mu^{\prime}
$$

Solutions for this form of the neutron transport equation are often found in the literature. The solution of this equation includes linearly anisotropic scattering; however, it also includes a linearly anisotropic fission 
source emission. For problems that include the anisotropic effect on the fission term, see Refs. 15,22,23. Solutions for the higher eigenvalues exist for this form of the transport equation. . $^{13,14,15,17,18,19,20}$

Numerical solutions exist that do not force the anisotropic effect on the fission term. This limitation on the different anisotropic behavior of scattering and fission can be removed by using different transfer functions for scattering and fission. The neutron transport equation can be written as: ${ }^{24}$

$$
\mu \frac{\partial \Psi(z, \mu)}{\partial z}+\Psi(z, \mu)=\frac{c}{2} \int_{-1}^{1} \Pi\left(\mu_{o}, \beta\right) \Psi\left(z, \mu^{\prime}\right) d \mu^{\prime}
$$

where:

$$
\begin{aligned}
\Pi\left(\mu_{o}, \beta\right) & =\beta \Pi_{s}\left(\mu_{o}\right)+(1-\beta) \Pi_{f}\left(\mu_{o}\right) \\
\beta & =\frac{\Sigma_{s}}{\Sigma_{s}+\nu \Sigma_{f}}, \quad 0 \leq \beta \leq 1
\end{aligned}
$$

The parameters, $\Pi_{s}\left(\mu_{o}\right)$ and $\Pi_{f}\left(\mu_{o}\right)$, are the angular transfer functions for scattering and fission, respectively $y_{\uparrow}$ and are dependent on $\mu$ and $\mu^{\prime}$. The weighting parameter, $\beta$, allows for the different anisotropic behavior for the scattering and fission terms. This is one of several ways found in the references of separating the anisotropic scattering and fission terms.

\section{Two-Energy Groups in One-Dimensional Slab Geometry}

1. Isotropic Scattering.

Using the same procedures as in the one-group case, the two-energy group form of the transport equation for a slab can be written as: ${ }^{8}$

$$
\begin{aligned}
& \mu \frac{\partial \Psi_{1}(x, \mu)}{\partial x}+\Sigma_{1} \Psi_{1}(x, \mu)=\frac{\Sigma_{11}}{2} \int_{-1}^{1} \Psi_{1}\left(x, \mu^{\prime}\right) d \mu^{\prime}+\frac{\Sigma_{12}}{2} \int_{-1}^{1} \Psi_{2}\left(x, \mu^{\prime}\right) d \mu^{\prime} \\
& \mu \frac{\partial \Psi_{2}(x, \mu)}{\partial x}+\Sigma_{2} \Psi_{2}(x, \mu)=\frac{\Sigma_{21}}{2} \int_{-1}^{1} \Psi_{1}\left(x, \mu^{\prime}\right) d \mu^{\prime}+\frac{\Sigma_{22}}{2} \int_{-1}^{1} \Psi_{2}\left(x, \mu^{\prime}\right) d \mu^{\prime}
\end{aligned}
$$

where:

$$
\begin{aligned}
\Sigma_{i}= & \text { total neutron macroscopic cross section of group } \mathrm{i} \\
\Sigma_{i j}= & \text { total neutron group transfer macroscopic cross section } \\
& \text { from group } \mathrm{j} \text { to group } \mathrm{i}
\end{aligned}
$$

In this report, the fast energy group is group 2 to be consistent with most of the references. This notation is the reverse of most nuclear engineering textbooks.

Assuming group 2 is the fast group and no nonfission up-scatter for the slow group 1, the group transfer cross sections are given by: 


$$
\begin{aligned}
& \Sigma_{11}=\Sigma_{11 s}+\chi_{1} \nu_{1} \Sigma_{1 f} / \mathrm{k}_{\mathrm{eff}} \\
& \Sigma_{21}=\chi_{2} \nu_{1} \Sigma_{1 f} / \mathrm{k}_{\mathrm{eff}} \\
& \Sigma_{12}=\Sigma_{12 s}+\chi_{1} \nu_{2} \Sigma_{2 f} / \mathrm{k}_{\mathrm{eff}} \\
& \Sigma_{22}=\Sigma_{22 s}+\chi_{2} \nu_{2} \Sigma_{2 f} / \mathrm{k}_{\mathrm{eff}}
\end{aligned}
$$

Note that $\Sigma_{i}=\Sigma_{i c}+\Sigma_{i f}+\Sigma_{i i s}+\Sigma_{j i s}$, where the $\Sigma_{j i s}$ represents nonfission scattering to group $j \neq i$. This equation for $\Sigma_{i}$ again assumes that the $\Sigma_{(n, 2 n)_{i}}+\ldots$ components are zero.

The two-energy group form of the transport equation, which has solutions in the literature, can be written in a similar form to the one-group equations utilizing the optical thickness parameter, $z=\Sigma_{2} x$, but in matrix-vector notation as seen below.

$$
\mu \frac{\partial \bar{\Psi}(z, \mu)}{\partial z}+\overline{\bar{\Sigma}} \bar{\Psi}(z, \mu)=\frac{\overline{\bar{C}}}{2} \int_{-1}^{1} \bar{\Psi}\left(z, \mu^{\prime}\right) d \mu^{\prime}
$$

where:

$$
\bar{\Psi}(z, \mu)=\left[\begin{array}{l}
\Psi_{1}(z, \mu) \\
\Psi_{2}(z, \mu)
\end{array}\right], \overline{\bar{\Sigma}}=\left[\begin{array}{cc}
\Sigma & 0 \\
0 & 1
\end{array}\right], \overline{\bar{C}}=\left[\begin{array}{ll}
c_{11} & c_{12} \\
c_{21} & c_{22}
\end{array}\right]
$$

and

$$
c_{i j}=\Sigma_{i j} / \Sigma_{2}
$$

The derivations for the infinite medium $\mathrm{k}_{\infty}$ and the group 2 to group 1 flux ratio are given in Appendix A.

\section{Linearly Anisotropic Scattering.}

One of the above simplifying assumptions to the steady state neutron transport equation is that neutron scattering is isotropic (no angular dependence). However, the scattering term, $\Sigma_{s}\left(\vec{r}, E^{\prime} \rightarrow E, \vec{\Omega}^{\prime} \rightarrow \vec{\Omega}\right.$ ) can be a strong function of the cosine of the scattering angle, $\mu_{0}=\vec{\Omega}^{\prime} \cdot \vec{\Omega}$. This angular dependence can be analyzed by Legendre polynomial series expansion of $\Sigma_{s}\left(\vec{r}, E^{\prime} \rightarrow E, \vec{\Omega}^{\prime} \cdot \vec{\Omega}\right),{ }^{21}$ giving

$$
\Sigma_{s}\left(\vec{r}, E^{\prime} \rightarrow E, \vec{\Omega}^{\prime} \cdot \vec{\Omega}\right)=\sum_{l=0}^{M} \frac{2 l+1}{4 \pi} \Sigma_{s l}\left(\vec{r}, E^{\prime} \rightarrow E\right) P_{l}\left(\vec{\Omega}^{\prime} \cdot \vec{\Omega}\right)
$$

where $M$ indicates the degree of anisotropy. For $M=0$, scattering in the lab system is isotropic and for $M=1$, scattering is linearly anisotropic. A complete mathematical description is in Ref. 12 and 21 . For linearly anisotropic scattering, the scattering cross section consists of two components, $\Sigma_{s_{0}}$ and $\Sigma_{s_{1}}$, where 
$\Sigma_{s_{1}}$ is the linear anisotropic scattering component and affects the scattering angular distribution for both in and out of group scattering. Anisotropic scattering can be forward or backward peaked and thus $\Sigma_{s_{1}}$ can be positive or negative. For linear anisotropic scattering in the one-energy group case, $\Sigma_{s}(\mu)=\left(\Sigma_{s_{0}}+3 \mu \Sigma_{s_{1}}\right) / 2$. Thus, $\Sigma_{s}(\mu)$ can become negative when $\left|\Sigma_{s_{1}}\right|>\Sigma_{s_{0}} / 3$. Test set problems $34,37,43$, and 71 exhibit this behavior. The total scattering cross section is not dependent on $\Sigma_{s_{1}}$. The anisotropic cross section only affects the angular distribution. Infinite medium $k_{\infty}$ and neutron flux results are independent of the anisotropic cross section.

Using the Legendre polynomial expansion, the neutron transport equation for a one-dimensional slab with azimuthally symmetric scattering can be written in a form similar to Eq. 2:

$$
\begin{aligned}
\mu \frac{\partial \Psi(x, E, \mu)}{\partial x}+\Sigma_{t}(x, E) \Psi(x, E, \mu)= & 2 \pi \int_{0}^{\infty} d E^{\prime} \sum_{l=0}^{M} \frac{2 l+1}{4 \pi} \Sigma_{s l}\left(E^{\prime} \rightarrow E\right) P_{l}(\mu) \int_{-1}^{1} P_{l}\left(\mu^{\prime}\right) \Psi\left(x, \mu^{\prime}\right) d \mu^{\prime} \\
& +\chi(E) \int_{0}^{\infty} d E^{\prime} \frac{\nu\left(x, E^{\prime}\right)}{2 \mathrm{k}_{\mathrm{eff}}} \Sigma_{f}\left(x, E^{\prime}\right) \int_{-1}^{1} \Psi\left(r, E^{\prime}, \mu^{\prime}\right) d \mu^{\prime}
\end{aligned}
$$

Following the same procedures as in Eq. 5, the general two-speed linearly anisotropically scattering analogue to Eq. 10 which also has numerical solutions is: ${ }^{25}$

$$
\mu \frac{\partial \bar{\Psi}(z, \mu)}{\partial z}+\overline{\bar{\Sigma}} \bar{\Psi}(z, \mu)=\frac{1}{2} \sum_{l=0}^{1} \overline{\bar{C}}_{l} P_{l}(\mu) \int_{-1}^{1} \bar{\Psi}\left(z, \mu^{\prime}\right) P_{l}\left(\mu^{\prime}\right) d \mu^{\prime}
$$

where:

$$
\bar{\Psi}(z, \mu)=\left[\begin{array}{l}
\Psi_{1}(z, \mu) \\
\Psi_{2}(z, \mu)
\end{array}\right], \bar{\Sigma}=\left[\begin{array}{cc}
\Sigma & 0 \\
0 & 1
\end{array}\right], \overline{\bar{C}}_{l}=\left[\begin{array}{ll}
c_{11 l} & c_{12 l} \\
c_{21 l} & c_{22 l}
\end{array}\right]
$$

and

$$
c_{i j l}=(2 l+1) \Sigma_{i j l} / \Sigma_{2}
$$

The $\Sigma_{i j s_{2}}$ term is the linearly anisotropic scattering cross section and is given in the problem descriptions without the $(2 l+1)$ term. 


\section{ONE-ENERGY GROUP PROBLEM DEFINITIONS AND RESULTS}

For the one-energy group cases, the critical dimension(s) for each geometry depends upon the $c$ value chosen from the literature and not specific cross-section sets. To use the literature results, the cross sections were selected to match published $c$ values with $\mathrm{k}_{e f f}=1$ at low, middle, and high $c$ values listed. Values ranging from $1.02,1.30,1.40$, and 1.50 were chosen because they are similar to the physical systems in the two-group cases: uranium- $\mathrm{D}_{2} \mathrm{O}$ reactor, $\mathrm{U}-235$, and $\mathrm{Pu}-239$. These problems use cross sections that are reasonable representations of these materials; however, these cross sections are not general purpose one-group values. The cross sections are used because they define the $c$ values used in the literature and are intended to be used only to verify algorithm performance and not to predict any actual criticality experiments.

The isotropic neutron macroscopic cross sections provided for each case are: the total cross section, $\Sigma_{t}$, the capture (no neutrons emitted) cross section, $\Sigma_{c}$, the scattering cross section, $\Sigma_{s}$, the fission cross section, $\Sigma_{f}$, and the number of neutrons, $\nu$, emitted for each fission. The $(n, 2 n),(n, 3 n), \ldots$ cross sections are assumed to be zero (but need not be). Thus the total cross section equals the sum of $\Sigma_{c}, \Sigma_{s}$, and $\Sigma_{f}$, thereby providing a consistency check on the cross-section set. Many references give $\left(\nu \Sigma_{f}\right)$ instead of $\nu$ and $\Sigma_{f}$. Since both parameters (not the product) may be required by a code for the problem solution, the product $\left(\nu \Sigma_{f}\right)$ has been split into $\nu$ and $\Sigma_{f}$ preserving their product and $\Sigma_{t}$. The value of $c$ for $k_{e f f}=1$ in Eq. 6 is also included in each cross-section table. For the reflected spheres, different secondaries ratios, $c$, are reported with the critical dimension for $\mathrm{k}_{e f f}=1$ for various combinations of core and reflector thicknesses. To maintain consistent cross sections with the U-235 set, the parameter, $\nu$, was modified to match $c$ to the literature values.

When anisotropic scattering cross sections are provided, the anisotropic components are designated by $\Sigma_{s_{1}}$ and $\Sigma_{s_{2}}$, respectively. Similarly, the isotropic scattering component is designated by $\Sigma_{s_{0}}$.

The value of $k_{\infty}$, as defined in Appendix A, is given for each cross-section set. For finite problems where $\mathbf{k}_{e f f}$ is unity, the critical dimension, $\mathbf{r}_{c}$, is listed for each geometry in both mean free paths (to indicate the neutron optical thickness) and in centimeters for the one-dimensional geometries. When available in the literature, the scalar flux values, normalized to the flux at the center of the fissile material, are also provided. The two-media problems have $c_{1}>1$ for the core region 1 and $c_{2}<1$ for the surrounding reflector region 2 . The two-media problems use the cross sections for the nonmultiplying reflector. The critical dimensions for the multiplying medium and reflector thickness are given in both mean free paths and centimeters.

A comparison of the critical dimensions for the different geometries behave as expected; that is, the critical dimension is smallest for the one-dimensional slab and increases for the cylinder and sphere. This behavior is to be expected due to the increased leakage with the curvi-linear geometries. For the reflected geometries, the critical dimension decreases with increasing reflector thickness. 


\section{A. One-Energy Group Isotropic Scattering}

\section{One-Group Pu-239.}

\section{One-Energy Group Isotropic Cross Sections}

Table 5 gives the one-group, isotropic cross sections for two case of $\mathrm{Pu}-239(\mathrm{c}=1.50$ and $\mathrm{c}=1.40)$ and a $\mathrm{H}_{2} \mathrm{O}(\mathrm{c}=0.90)$ reflector. The total cross sections are the same for both $\mathrm{Pu}-239$ cases and $\mathrm{H}_{2} \mathrm{O}$ as required by the reference for the two-media solutions.

Table 5: One-Group Macroscopic Cross Sections $\left(\mathrm{cm}^{-1}\right)$ for $\mathrm{Pu}-239(\mathrm{c}=1.40,1.50)$ and $\mathrm{H}_{2} \mathrm{O}(\mathrm{c}=0.90)$

\begin{tabular}{|c|c|c|c|c|c|c|}
\hline Material & $\nu$ & $\Sigma_{f}$ & $\Sigma_{c}$ & $\Sigma_{s}$ & $\Sigma_{t}$ & $\mathrm{c}$ \\
\hline $\mathrm{Pu}-239(\mathrm{a})$ & 3.24 & 0.081600 & 0.019584 & 0.225216 & 0.32640 & 1.50 \\
$\mathrm{Pu}-239(\mathrm{~b})$ & 2.84 & 0.081600 & 0.019584 & 0.225216 & 0.32640 & 1.40 \\
$\mathrm{H}_{2} \mathrm{O}$ (reff) & 0.0 & 0.0 & 0.032640 & 0.293760 & 0.32640 & 0.90 \\
\hline
\end{tabular}

Infinite Medium (PUa-1-0-IN and PUb-1-0-IN)

Using the cross sections for $\mathrm{Pu}-239$ (a) (problem 1) in Table 5, $\mathrm{k}_{\infty}=2.612903$ with a constant angular and scalar flux everywhere. Using the cross sections for Pu-239 (b) (problem 5) in Table 5, $\mathrm{k}_{\infty}=2.290323$ with a constant angular and scalar flux everywhere.

One-Medium Slab, Cylinder, and Sphere Critical Dimensions

The Pu-239 (a) critical dimension, $\mathbf{r}_{c}$, is listed in Table 6.

Table 6: Critical Dimensions, $\mathrm{r}_{c}$, for One-Group Bare Pu-239 (c=1.50)

\begin{tabular}{|c|c|c|c|c|c|}
\hline Problem & Identifier & Geometry & $\mathrm{r}_{c}(\mathrm{mfp})$ & $\mathrm{r}_{c}(\mathrm{~cm})$ & Reference \\
\hline 2 & PUa-1-0-SL & Slab & 0.605055 & 1.853722 & 9 \\
\hline
\end{tabular}

The Pu-239 (b) critical dimensions, $\mathrm{r}_{c}$, are listed in Table 7. The normalized scalar flux for four spatial positions are given in Table 8 using the same references. The flux ratios for PUb-1-0-CY are only available to four decimal places.

Table 7: Critical Dimensions, $\mathrm{r}_{c}$, for One-Group Bare Pu-239 ( $\left.c=1.40\right)$

\begin{tabular}{|c|c|c|c|c|c|}
\hline Problem & Identifier & Geometry & $\mathrm{r}_{c}(\mathbf{m f p})$ & $\mathrm{r}_{c}(\mathbf{c m})$ & Reference \\
\hline 6 & PUb-1-0-SL & Slab & 0.73660355 & 2.256751 & 26 \\
7 & PUb-1-0-CY & Cylinder & 1.396979 & 4.279960 & 27,28 \\
8 & PUb-1-0-SP & Sphere & 1.9853434324 & 6.082547 & 26 \\
\hline
\end{tabular}

Table 8: Normalized Scalar Fluxes for One-Group Bare Pu-239 ( $c=1.40)$

\begin{tabular}{|c|c|c|c|c|c|c|}
\hline Problem & Identifier & Geometry & $\mathbf{r} / \mathbf{r}_{c}=0.25$ & $\mathbf{r} / \mathrm{r}_{c}=0.5$ & $\mathrm{r} / \mathbf{r}_{c}=0.75$ & $\mathrm{r} / \mathrm{r}_{c}=1.0$ \\
\hline 6 & PUb-1-0-SL & Slab & 0.9701734 & 0.8810540 & 0.7318131 & 0.4902592 \\
7 & PUb-1-0-CY & Cylinder & - & 0.8093 & - & 0.2926 \\
8 & PUb-1-0-SP & Sphere & 0.93538006 & 0.75575352 & 0.49884364 & 0.19222603 \\
\hline
\end{tabular}


Two-Media Slab and Cylinder Critical Dimensions

The literature values in Tables 9 and 10, give the critical dimensions for $\mathrm{Pu}-239$ (a) for two $\mathrm{H}_{2} \mathrm{O}$ reflector thicknesses. The first two-media problem (problem 3) in Table 9 is a special nonsymmetric two-region, $\mathrm{Pu}-$ 239 and $\mathrm{H}_{2} \mathrm{O}$, problem. The second two-media problem (problem 4) in Table 10 is a symmetric three-region problem with the reflector on both sides of the fissile medium.

Table 9: Critical Dimensions for One-Group Pu-239 Slab (c=1.50) with Non-Symmetric $\mathrm{H}_{2} \mathrm{O}$ Reflector $(\mathrm{c}=0.90)$

\begin{tabular}{|c|c|c|c|c|c|c|}
\hline Problem & Identifier & Geometry & ${\mathrm{Pu} \mathrm{r}_{c}}$ & $\mathrm{H}_{2} \mathrm{O}$ thickness & Pu $+\mathrm{H}_{2} \mathrm{O}$ Radius & Reference \\
\hline 3 & PUa-H2O(1)-1-0-SL & Slab (mfp) & 0.48255 & 1 & & 9 \\
& & $(\mathrm{~cm})$ & 1.478401 & 3.063725 & 4.542126 & \\
\hline
\end{tabular}

Table 10: Critical Dimensions for One-Group Pu-239 Slab ( $c=1.50)$ with $\mathrm{H}_{2} \mathrm{O}$ Reflector $(c=0.90)$

\begin{tabular}{|c|c|c|c|c|c|c|}
\hline Problem & Identifier & Geometry & $\mathrm{Pu}_{c}$ & $\mathrm{H}_{2} \mathrm{O}$ thickness & $\mathrm{Pu}+\mathrm{H}_{2} \mathrm{O}$ radius & Reference \\
\hline 4 & PUa-H2O(0.5)-1-0-SL & $\begin{array}{r}\text { Slab (mfp) } \\
(\mathrm{H} 2 \mathrm{O})\end{array}$ & $\begin{array}{c}0.43014 \\
1.317831\end{array}$ & $\begin{array}{c}0.5 \\
1.531863\end{array}$ & 2.849694 & 9 \\
\hline
\end{tabular}

The literature values in Table 11, give the critical dimensions for $\mathrm{Pu}-239$ (b) with two $\mathrm{H}_{2} \mathrm{O}$ reflector thicknesses.

Table 11: Critical Dimensions for One-Group Pu-239 Cylinder (c=1.40) with $\mathrm{H}_{2} \mathrm{O}$ Reflector $(c=0.90)$

\begin{tabular}{|c|c|c|c|c|c|c|}
\hline Problem & Identifier & Geometry & $\mathrm{Pu} \mathrm{r}_{\boldsymbol{c}}$ & $\mathrm{H}_{2} \mathrm{O}$ thickness & $\mathrm{Pu}+\mathrm{H}_{2} \mathrm{O}$ Radius & Reference \\
\hline 9 & PUb-H2O(1)-1-0-CY & $\begin{array}{r}\text { Cylinder (mfp) } \\
(\mathrm{cm})\end{array}$ & $\begin{array}{c}1.10898 \\
3.397610\end{array}$ & $\begin{array}{c}1 \\
3.063725\end{array}$ & 6.461335 & 29 \\
\hline 10 & PUb-H2O(10)-1-0-CY & $\begin{array}{r}\text { Cylinder (mfp) } \\
(\mathrm{cm})\end{array}$ & $\begin{array}{c}1.00452 \\
3.077574\end{array}$ & $\begin{array}{c}10 \\
30.637255\end{array}$ & 33.714829 & 29 \\
\hline
\end{tabular}




\section{One-Group U-235.}

\section{One-Group Isotropic Cross Sections}

Table 12 gives the one-group, isotropic cross sections for two cases of $\mathrm{U}-235$ and a $\mathrm{H}_{2} \mathrm{O}$ reflector. Notice that one-group $\Sigma_{t}$ for Pu-239 and U-235 are the same as given in reference 32, but the secondaries ratio, $c$, differs.

Table 12: One-Group Macroscopic Cross Sections $\left(\mathrm{cm}^{-1}\right)$ for U-235 $(c=1.30)$

\begin{tabular}{|c|c|c|c|c|c|c|}
\hline Material & $\nu$ & $\Sigma_{f}$ & $\Sigma_{c}$ & $\Sigma_{s}$ & $\Sigma_{t}$ & $\mathrm{c}$ \\
\hline $\mathrm{U}-235$ (a) & 2.70 & 0.065280 & 0.013056 & 0.248064 & 0.32640 & 1.30 \\
$\mathrm{U}-235$ (b) & 2.797101 & 0.065280 & 0.013056 & 0.248064 & 0.32640 & 1.3194202 \\
$\mathrm{U}-235$ (c) & 2.707308 & 0.065280 & 0.013056 & 0.248064 & 0.32640 & 1.3014616 \\
$\mathrm{U}-235$ (d) & 2.679198 & 0.065280 & 0.013056 & 0.248064 & 0.32640 & 1.2958396 \\
$\mathrm{H}_{2} \mathrm{O}$ (refl) & 0.0 & 0.0 & 0.032640 & 0.293760 & 0.32640 & 0.90 \\
\hline
\end{tabular}

Infinite Medium (Ua-1-0-IN , Ub-1-0-IN , Uc-1-0-IN , and Ud-1-0-IN )

Using the cross sections for U-235 (a) in Table $12, \mathrm{k}_{\infty}=2.25$ (problem 11) with a constant angular and scalar flux everywhere. Using the cross sections for U-235 (b), U-235 (c), and U-235 (d) in Table 12, $\mathrm{k}_{\infty}$ $=2.330917$ (problem 15), 2.256083 (problem 17), and 2.232667 (problem 19) with a constant angular and scalar flux everywhere, respectively.

One-Medium Slab, Cylinder, and Sphere Critical Dimensions

The critical dimension, $r_{c}$, and spatial flux ratios are given in Table 13 and 14 for U-235 (a). The references are the same for both tables.

Table 13: Critical Dimensions, $\mathbf{r}_{c}$, for One-Group Bare U-235 ( $\left.c=1.30\right)$

\begin{tabular}{|c|c|c|c|c|c|}
\hline Problem & Identifier & Geometry & $\mathbf{r}_{c}(\mathrm{mfp})$ & $\mathbf{r}_{c}(\mathbf{c m})$ & Reference \\
\hline 12 & Ua-1-0-SL & Slab & 0.93772556 & 2.872934 & 26 \\
13 & Ua-1-0-CY & Cylinder & 1.72500292 & 5.284935 & 27,28 \\
14 & Ua-1-0-SP & Sphere & 2.4248249802 & 7.428998 & 26 \\
\hline
\end{tabular}

Table 14: Normalized Scalar Fluxes for One-Group Bare U-235 $(c=1.30)$

\begin{tabular}{|c|c|c|c|c|c|c|}
\hline Problem & Identifier & Geometry & $\mathrm{r} / \mathrm{r}_{c}=0.25$ & $\mathrm{r} / \mathrm{r}_{c}=0.5$ & $\mathrm{r} / \mathrm{r}_{c}=0.75$ & $\mathrm{r} / \mathrm{r}_{c}=1.0$ \\
\hline 12 & Ua-1-0-SL & Slab & 0.9669506 & 0.8686259 & 0.7055218 & 0.4461912 \\
14 & Ua-1-0-SP & Sphere & 0.93244907 & 0.74553332 & $\mathbf{0 . 4 8 0 9 5 4 1 3}$ & $\mathbf{0 . 1 7 1 7 7 7 0 6}$ \\
\hline
\end{tabular}


Two-Media Sphere Critical Dimensions

The literature values in Table 15, give the critical dimensions for U-235 (b), U-235 (c), and U-235 (d) for three spherical $\mathrm{H}_{2} \mathrm{O}$ reflector thicknesses.

Table 15: Critical Dimensions for One-Group U-235 Sphere with $\mathrm{H}_{2} \mathrm{O}$ Reflector (c=0.90)

\begin{tabular}{|c|c|c|c|c|c|c|}
\hline Problem & Identifier & Geometry & $\mathrm{U}_{c}$ & $\mathrm{H}_{2} \mathrm{O}$ thickness & $\mathrm{U}^{+} \mathrm{H}_{2} \mathrm{O}$ Radius & Reference \\
\hline 16 & $\mathrm{Ub}-\mathrm{H} 2 \mathrm{O}(1)-1-0-\mathrm{SP}$ & Sphere (mfp) & 2 & 1 & & 17,20 \\
& & $(\mathrm{~cm})$ & 6.12745 & 3.063725 & 9.191176 & \\
18 & $\mathrm{Uc-H} 2 \mathrm{O}(2)-1-0-\mathrm{SP}$ & Sphere (mf) & 2 & 2 & & 17,20 \\
& & $(\mathrm{~cm})$ & 6.12745 & 6.12745 & 12.2549 & \\
20 & $\mathrm{Ud}-\mathrm{H} 2 \mathrm{O}(3)-1-0-\mathrm{SP}$ & Sphere (mf) & 2 & 3 & & 17,20 \\
& & $(\mathrm{~cm})$ & 6.12745 & 9.191176 & 15.318626 & \\
\hline
\end{tabular}




\section{One-Group U- $\mathrm{D}_{2} \mathrm{O}$ Reactor.}

One-Group Isotropic Cross Sections

Table 16 gives the one-group, isotropic cross sections for the uranium- $\mathrm{D}_{2} \mathrm{O}$ reactor and $\mathrm{H}_{2} \mathrm{O}$ reflector. Note that the uranium- $\mathrm{D}_{2} \mathrm{O}$ reactor and $\mathrm{H}_{2} \mathrm{O}$ reflector have the same total cross section as required by the references for the reflected cylindrical solutions.

Table 16: One-Group Macroscopic Cross Sections $\left(\mathrm{cm}^{-1}\right)$ for $U-\mathrm{D}_{2} \mathrm{O}$ Reactor $(\mathrm{c}=1.02)$ and $\mathrm{H}_{2} \mathrm{O}(\mathrm{c}=0.90)$

\begin{tabular}{|c|c|c|c|c|c|c|}
\hline Material & $\nu$ & $\Sigma_{f}$ & $\Sigma_{c}$ & $\Sigma_{s}$ & $\Sigma_{t}$ & $c$ \\
\hline $\mathrm{U}_{2} \mathrm{D}_{2} \mathrm{O}$ & 1.70 & 0.054628 & 0.027314 & 0.464338 & 0.54628 & 1.02 \\
$\mathrm{H}_{2} \mathrm{O}$ (refl) & 0.0 & 0.0 & 0.054628 & 0.491652 & 0.54628 & 0.90 \\
\hline
\end{tabular}

Infinite Medium (UD2O-1-0-IN)

Using the cross sections for $U-\mathrm{D}_{2} \mathrm{O}$ in Table $16, \mathrm{k}_{\infty}=1.133333$ (problem 21) with a constant angular and scalar flux everywhere.

One-Medium Slab, Cylinder, and Sphere Critical Dimensions

The critical dimension, $\mathrm{r}_{\boldsymbol{c}}$, and spatial flux ratios are listed in Table 17 and 18.

Table 17: Critical Dimensions, $\mathrm{r}_{c}$, for One-Group Bare $\mathrm{U}-\mathrm{D}_{2} \mathrm{O}$ Reactor $(\mathrm{c}=1.02)$

\begin{tabular}{|c|c|c|c|c|c|}
\hline Problem & Identifier & Geometry & $\mathrm{r}_{c}(\mathrm{mfp})$ & $\mathrm{r}_{c}(\mathrm{~cm})$ & Reference \\
\hline 22 & UD2O-1-0-SL & Slab & 5.6655054562 & 10.371065 & 26 \\
23 & UD2O-1-0-CY & Cylinder & 9.043255 & 16.554249 & 27,28 \\
24 & UD2O-1-0-SP & Sphere & 12.0275320980 & 22.017156 & 26 \\
\hline
\end{tabular}

Table 18: Normalized Scalar Fluxes for One-Group Bare U- $\mathrm{D}_{2} \mathrm{O}$ Reactor $(\mathrm{c}=1.02)$

\begin{tabular}{|c|c|c|c|c|c|c|}
\hline Problem & Identifier & Geometry & $\mathrm{r} / \mathrm{r}_{c}=0.25$ & $\mathrm{r} / \mathrm{r}_{c}=0.5$ & $\mathrm{r} / \mathrm{r}_{c}=0.75$ & $\mathrm{r} / \mathrm{r}_{c}=1.0$ \\
\hline 22 & UD2O-1-0-SL & Slab & 0.93945236 & 0.76504084 & 0.49690627 & 0.13893858 \\
24 & UD2O-1-0-SP & Sphere & 0.91063756 & 0.67099621 & 0.35561622 & 0.04678614 \\
\hline
\end{tabular}

Two-Media Slabs and Cylinders Critical Dimensions

Table 19 gives the U- $\mathrm{D}_{2} \mathrm{O}$ critical dimension, $\mathrm{r}_{c}$, for two $\mathrm{H}_{2} \mathrm{O}$ reflector thicknesses.

Table 19: Critical Dimensions for One-Group $\mathrm{U}_{-} \mathrm{D}_{2} \mathrm{O}(\mathrm{c}=1.02)$ Slab and Cylinder with $\mathrm{H}_{2} \mathrm{O}(\mathrm{c}=0.90)$ Reflector

\begin{tabular}{|c|c|c|c|c|c|c|}
\hline Problem & Identifier & Geometry & $\mathrm{UD}_{2}{\mathrm{O} \mathrm{r}_{c}}_{\mathrm{s}}$ & $\mathrm{H}_{2} \mathrm{O}$ thickness & $\mathrm{UD}_{2} \mathrm{O}+\mathrm{H}_{2} \mathrm{O}$ radius & Reference \\
\hline 25 & UD2O-H2O(1)-1-0-SL & $\begin{array}{c}\text { Slab (mfp) } \\
(\mathrm{cm})\end{array}$ & $\begin{array}{c}5.0335 \\
9.214139\end{array}$ & $\begin{array}{c}1 \\
1.830563\end{array}$ & 11.044702 & 33,34 \\
\hline 26 & UD2O-H2O(10)-1-0-SL & $\begin{array}{r}\text { Slab (mfp) } \\
(\mathrm{cm})\end{array}$ & $\begin{array}{c}4.6041 \\
8.428096\end{array}$ & $\begin{array}{c}10 \\
18.30563\end{array}$ & 26.733726 & 33,34 \\
\hline 27 & $\mathrm{UD} 2 \mathrm{O}-\mathrm{H} 2 \mathrm{O}(1)-1-0-\mathrm{CY}$ & $\begin{array}{r}\text { Cylinder (mfp) } \\
(\mathrm{cm})\end{array}$ & $\begin{array}{c}8.411027 \\
15.396916\end{array}$ & $\begin{array}{c}1 \\
1.830563\end{array}$ & 17.227479 & 29 \\
\hline 28 & UD2O-H2O(10)-1-0-CY & $\begin{array}{r}\text { Cylinder (mfp) } \\
(\mathrm{cm})\end{array}$ & $\begin{array}{c}7.979325 \\
14.606658\end{array}$ & $\begin{array}{c}10 \\
18.30563\end{array}$ & 32.912288 & 29 \\
\hline
\end{tabular}




\section{One-Group U-235 Reactor.}

\section{One-Group Isotropic Cross Sections}

Table 20 gives the one-group, isotropic cross sections for the U-235 reactor with a Fe reflector and $\mathrm{Na}$ moderator.

Table 20: One-Group Macroscopic Cross Sections $\left(\mathrm{cm}^{-1}\right)$ for U-235 Reactor, Fe reflector, and Na Moderator

\begin{tabular}{|c|c|c|c|c|c|c|}
\hline Material & $\nu$ & $\Sigma_{f}$ & $\Sigma_{c}$ & $\Sigma_{s}$ & $\Sigma_{t}$ & $\mathrm{c}$ \\
\hline $\mathrm{U}-235$ (e) & 2.50 & 0.06922744 & 0.01013756 & 0.328042 & 0.407407 & 1.230 \\
$\mathrm{Fe}$ (ref) & 0.0 & 0.0 & 0.00046512 & 0.23209488 & 0.23256 & 0.9980 \\
$\mathrm{Na}$ (mod) & 0.0 & 0.0 & 0.0 & 0.086368032 & 0.086368032 & 1.00 \\
\hline
\end{tabular}

Infinite Medium (Ue-1-0-IN)

Using the cross sections for the U-235 reactor in Table $20, \mathrm{k}_{\infty}=2.1806667$ (problem 29) with a constant angular and scalar flux everywhere.

\section{One-Medium Slab Critical Dimensions}

Note that this problem is a nonsymmetric four-region problem. The U-235 is surrounded by a Fe cladding on two sides but moderated by $\mathrm{Na}$ on one side. The critical dimension, $\mathrm{r}_{e}$, is listed in Tables 21 and 22 .

Table 21: Critical Dimensions, $\mathbf{r}_{c}$, for One-Group U-235 Reactor

\begin{tabular}{|c|c|c|c|c|c|c|c|}
\hline Problem & Identifier & Geometry & Fe thickness & U-235 thickness & Fe thickness & Na thickness & Reference \\
\hline 30 & Ue-Fe-Na-1-0-SL & Slab (mfp) & 0.0738 & 2.0858098 & 0.0738 & 0.173 & 45 \\
& & $(\mathrm{~cm})$ & 0.317337461 & 5.119720083 & 0.317337461 & 2.002771002 & \\
\hline
\end{tabular}

Table 22: Critical Dimensions, $\mathrm{r}_{c}$, for One-Group U-235 Reactor

\begin{tabular}{|c|c|c|c|c|c|c|}
\hline Problem & Identifier & Geometry & Fe thickness & Fe+U & Fe+U+Fe & Fe+U+Fe+Na \\
\hline 30 & Ue-Fe-Na-1-0-SL & Slab $(\mathrm{cm})$ & 0.317337461 & 5.437057544 & 5.754395005 & 7.757166007 \\
\hline
\end{tabular}

The U-235 (e) critical dimensions, $r_{c}$, are listed in Tables 21 and 22 . The normalized scalar flux for four spatial positions are given in Table 23 using the same references. These positions correspond to the material boundaries and are normalized by the scalar neutron flux at the left boundary.

Table 23: Normalized Scalar Fluxes for One-Group U-235 Reactor

\begin{tabular}{|c|c|c|c|c|c|c|}
\hline Problem & Identifier & Geometry & Fe-U & U-Fe & Fe-Na & $\mathrm{Na}$ \\
\hline 30 & Ue-Fe-Na-1-0-SL & Slab & 1.229538 & 1.49712 & 1.324899 & 0.912273 \\
\hline
\end{tabular}




\section{B. One-Group Anisotropic Scattering}

\section{One-Group Pu-239.}

\section{One-Energy Group Anisotropic Cross Sections}

Table 24 gives the one-group, anisotropic cross sections for two cases of anisotropic scattering. The first cross-section set, $\mathrm{Pu}-239$ (a), includes $P_{1}$ and $P_{2}$ scattering cross sections, where $|\mu|<1 / 3$. The second cross-section set, $\mathrm{Pu}-239$ (b), includes the $\mathrm{P}_{1}$ and $\mathrm{P}_{2}$ scattering cross sections where $|\mu|>1 / 3$. Care must be used to correctly solve benchmark problem 34 because of the negative scattering for $\mu$ near -1 .

Table 24: One-Group Macroscopic Anisotropic Cross Sections $\left(\mathrm{cm}^{-1}\right)$ for Pu-239 $(\mathrm{c}=1.40)$

\begin{tabular}{|c|c|c|c|c|c|c|c|c|}
\hline Material & $\nu$ & $\Sigma_{f}$ & $\Sigma_{c}$ & $\Sigma_{s_{0}}$ & $\Sigma_{s_{1}}$ & $\Sigma_{s_{2}}$ & $\Sigma_{t}$ & $\mathrm{c}$ \\
\hline $\mathrm{Pu}-239(\mathrm{a})$ & 2.5 & 0.266667 & 0.0 & 0.733333 & 0.20 & 0.075 & 1.0 & 1.40 \\
$\mathrm{Pu}-239(\mathrm{~b})$ & 2.5 & 0.266667 & 0.0 & 0.733333 & 0.333333 & 0.125 & 1.0 & 1.40 \\
\hline
\end{tabular}

Infinite Medium (PU-1-1-IN)

Using the cross sections for Pu-239 (a) and Pu-239 (b) in Table 24, $\mathrm{k}_{\infty}=2.5$ (problem 31) with a constant angular and scalar flux everywhere. The anisotropic scattering cross sections do not change $\mathbf{k}_{\infty}$.

One-Medium Slab Critical Dimensions

The $\mathrm{Pu}-239$ critical dimensions, $\mathrm{r}_{c}$, for both $\mathrm{P}_{1}$ and $\mathrm{P}_{2}$ problems are listed in Table 25 .

Table 25: Critical Dimensions, $\mathrm{r}_{c}$, for One-Group Bare Pu-239 ( $\left.\mathrm{c}=1.40\right)$

\begin{tabular}{|c|c|c|c|c|c|}
\hline Problem & Identifier & Geometry & $\mathbf{r}_{c}(\mathrm{mfp})$ & $\mathbf{r}_{c}(\mathrm{~cm})$ & Reference \\
\hline 32 & PUa-1-1-SL & Slab & 0.77032 & 0.77032 & 30 \\
33 & PUa-1-2-SL & Slab & 0.76378 & 0.76378 & 30 \\
34 & PUb-1-1-SL & Slab & 0.79606 & 0.79606 & 30 \\
35 & PUb-1-2-SL & Slab & 0.78396 & 0.78396 & 30 \\
\hline
\end{tabular}




\section{One-Group U-235}

\section{One-Energy Group Anisotropic Cross Sections}

Table 26 gives the two sets of one-group, anisotropic cross sections for U-235. Notice that the cross sections are the same as in Table 12 with the addition of $P_{1}$ scattering cross sections. The first cross-section set, U-235 (a), includes $P_{1}$ scattering cross sections, where $|\mu|<1 / 3$. The second cross-section set, U-235 (b), includes the $\mathrm{P}_{1}$ scattering cross sections where $|\mu|>1 / 3$. Care must be used to correctly solve benchmark problem 37 because of the negative scattering for $\mu$ near -1 .

Table 26: One-Group Macroscopic Anisotropic Cross Sections $\left(\mathrm{cm}^{-1}\right)$ for U-235 (c=1.30)

\begin{tabular}{|c|c|c|c|c|c|c|c|}
\hline Material & $\nu$ & $\Sigma_{f}$ & $\Sigma_{c}$ & $\Sigma_{s_{0}}$ & $\Sigma_{s_{1}}$ & $\Sigma_{t}$ & $c$ \\
\hline U-235 (a) & 2.70 & 0.065280 & 0.013056 & 0.248064 & 0.042432 & 0.32640 & 1.30 \\
U-235 (b) & 2.70 & 0.065280 & 0.013056 & 0.248064 & 0.212160 & 0.32640 & 1.30 \\
\hline
\end{tabular}

Infinite Medium (U-1-1-IN)

Using the cross sections for U-235 (a) and U-235 (b) in Table $26, \mathrm{k}_{\infty}=2.25$ (problem 11) with a constant angular and scalar flux everywhere. The anisotropic scattering cross sections do not change $k_{\infty}$.

One-Medium Slab Critical Dimensions

The U-235 critical dimensions, $\mathrm{r}_{c}$, for both $\mathrm{P}_{1}$ problems are listed in Table 27.

Table 27: Critical Dimensions, $r_{c}$, for One-Group Bare U-235 ( $\left.c=1.30\right)$

\begin{tabular}{|c|c|c|c|c|c|}
\hline Problem & Identifier & Geometry & $\mathrm{r}_{c}(\mathrm{mfp})$ & $\mathrm{r}_{c}(\mathrm{~cm})$ & Reference \\
\hline 36 & Ua-1-1-CY & Cylinder & 1.799866479 & 5.514296811 & 31 \\
37 & Ub-1-1-CY & Cylinder & 2.265283130 & 6.940205668 & 31 \\
\hline
\end{tabular}




\section{One-Group U- $\mathrm{D}_{2} \mathrm{O}$}

\section{One-Energy Group Anisotropic Cross Sections}

Table 28 gives the two sets of one-group, anisotropic cross sections for $U-\mathrm{D}_{2} \mathrm{O}$ reactor. Notice that the cross sections are the same as in Table 16 with the addition of $P_{1}$ scattering cross sections. The cross-ections set for two $U-D_{2} O$ cases include $P_{1}$ scattering cross sections, where $|\mu|<1 / 3$, and a $P_{1}$ case where $\mu<0$ and the scattering cross section is negative. Care must be used to correctly solve benchmark problem 43 because of the negative scattering for $\mu$ near 1 .

Table 28: One-Group Macroscopic Anisotropic Cross Sections $\left(\mathrm{cm}^{-1}\right)$ for $\mathrm{U}_{-} \mathrm{D}_{2} \mathrm{O}$ Reactor

\begin{tabular}{|c|c|c|c|c|c|c|c|}
\hline Material & $\nu$ & $\Sigma_{f}$ & $\Sigma_{c}$ & $\Sigma_{s_{0}}$ & $\Sigma_{s_{1}}$ & $\Sigma_{t}$ & $\mathrm{c}$ \\
\hline $\mathrm{U}-\mathrm{D}_{2} \mathrm{O}(\mathrm{a})$ & 1.808381 & 0.054628 & 0.027314 & 0.464338 & 0.056312624 & 0.54628 & 1.0308381 \\
$\mathrm{U}-\mathrm{D}_{2} \mathrm{O}(\mathrm{b})$ & 1.841086 & 0.054628 & 0.027314 & 0.464338 & 0.112982569 & 0.54628 & 1.0341086 \\
$\mathrm{U}-\mathrm{D}_{2} \mathrm{O}(\mathrm{c})$ & 1.6964 & 0.054628 & 0.027314 & 0.464338 & -0.27850447 & 0.54628 & 1.01964 \\
\hline
\end{tabular}

Infinite Medium UD2Oa-1-1-IN , UD2Ob-1-1-IN , and UD2Oc-1-1-IN

Using the cross sections for $U-\mathrm{D}_{2} \mathrm{O}(\mathrm{a}), \mathrm{U}-\mathrm{D}_{2} \mathrm{O}(\mathrm{b})$, and $\mathrm{U}-\mathrm{D}_{2} \mathrm{O}(\mathrm{c})$ in Table $28, \mathrm{k}_{\infty}=1.205587$ (problem 38 ), 1.227391 (problem 40), and 1.130933 (problem 42), respectively, with a constant angular and scalar flux everywhere. The anisotropic scattering cross sections do not change $\mathrm{k}_{\infty}$.

One-Medium Slab Critical Dimensions

The U- $\mathrm{D}_{2} \mathrm{O}$ critical dimensions, $r_{c}$, for the $\mathrm{P}_{1}$ problems are listed in Table 29.

Table 29: Critical Dimensions, $\mathrm{r}_{c}$, for One-Group Bare U- $\mathrm{D}_{2} \mathrm{O}$

\begin{tabular}{|c|c|c|c|c|c|}
\hline Problem & Identifier & Geometry & $\mathbf{r}_{c}(\mathrm{mfp})$ & $\mathbf{r}_{c}(\mathrm{~cm})$ & Reference \\
\hline 39 & UD2Oa-1-1-SP & Sphere & 10 & 18.30563081 & 15 \\
41 & UD2Ob-1-1-SP & Sphere & 10 & 18.30563081 & 15 \\
43 & UD2Oc-1-1-SP & Sphere & 10 & 18.30563081 & 16 \\
\hline
\end{tabular}




\section{TWO-ENERGY GROUP PROBLEM DEFINITIONS}

\section{AND RESULTS}

The isotropic two-energy group cross sections for five bare and two water reflected cases are listed in this section. There are also two linearly anisotropic scattering cross-sections sets provided for bare and infinite medium reactors. Unlike the one-group case, there is no flexibility in choosing these values since they are used throughout the literature. The cross sections listed here are similar to $\mathrm{Pu}-239,{ }^{32} \mathrm{U}-235,{ }^{32}$ a realistic enriched uranium-aluminum-water assembly, ${ }^{8}$ a $93 \%$ enriched U-235 model of a university research reactor, ${ }^{8,35,36}$ and a typical large size $D_{2} \mathrm{O}$ reactor with low enrichment of $U-235{ }^{8,35,36}$ Also included are critical dimensions for a similar uranium research reactor with a water reflector in an infinite lattice. ${ }^{34}$ Again, these problems use cross sections that are reasonable representations of the materials described. These cross sections are not general purpose two-group values. The cross sections are used because they are defined in the literature and are intended to be used only to verify algorithm performance and not to predict any actual criticality experiments.

The isotropic neutron cross macroscopic sections $\left(\mathrm{cm}^{-1}\right)$ provided for these problems are the total cross section of group $i, \Sigma_{i}$, the capture (no neutrons emitted) cross section, $\Sigma_{c i}$, the within group scattering cross section, $\Sigma_{i i s}$, the group-to-group scattering cross sections, $\Sigma_{i j s}$ and $\Sigma_{j i s}$, the fission cross section, $\Sigma_{i f}$, the number of neutrons, $\nu_{i}$, emitted from each fission in a group, and the fission distribution, $\chi_{i}$.

In this report, the fast energy group is group 2 to be consistent with most of the references. This notation is the reverse of most nuclear engineering textbooks.

The literature solutions are often based on the group transfer cross sections, $\Sigma_{i j}$, given in the references; therefore, the individual cross sections may not be unique. Most references give $\left(\nu \Sigma_{f}\right)_{i}$ instead of $\nu_{i}$ and $\Sigma_{f i}$. Since both parameters (not the product) may be required by a code for the problem solution, the product $\left(\nu \Sigma_{f}\right)_{i}$ has been split into $\nu_{i}$ and $\Sigma_{f i}$ preserving their product and $\Sigma_{t}$. The infinite slab lattice problems use a slightly unphysical set of cross sections to possibly stress code verification.

The two sets of linearly anisotropic cross sections provided are extensions of the university research reactor and $\mathrm{D}_{2} \mathrm{O}$ cases. ${ }^{25}$ The anisotropic scattering component is designated for the in-group and group-togroup scattering cross section by $\Sigma_{i s_{1}}$ and $\Sigma_{j i s_{1}}$, respectively. Similarly, the isotropic scattering component is designated by $\Sigma_{i i_{0}}$ and $\Sigma_{j i s_{0}}$.

The value for $k_{\infty}$ is given for each cross-section set. The in-group scattering cross sections do not affect $\mathbf{k}_{\infty}$. For finite problems, the critical dimension, $\mathrm{r}_{c}$, is listed in both fast group mean free paths (to indicate the neutron optical thickness) and in centimeters. For two-media problems, critical dimensions for the inner multiplying medium and outer reflector thickness are given in both fast mean free paths and centimeters. The critical dimensions, $r_{c}$, and reflector half thicknesses are also given for a water reflected infinite slab 
lattice cell. Flux values are given for the university research reactor (a) (problems 54 and 71) at four spatial points. Angular fluxes can be found in the dissertation references.

To distinguish between the different URR fissile material cross-section sets, each is labeled with a letter " $a, "$ " $b$, ," $c$," or " $d$ ", respectively. The infinite slab lattice cell cross sections are similar to the other three cross-section sets for the university research reactor and are labeled with URRd identifiers. The literature also uses three different $\mathrm{H}_{2} \mathrm{O}$ reflectors. Their cross sections are also labeled with a letter " $a$," " $b$," or " $c$ " in the identifier. URR cross-section sets " $b$ " and " $c$ " have thermal upscattering. All other two-group cross sections have no thermal upscattering.

A comparison of the critical dimensions for the different geometries behave as expected; that is, the critical dimension is smallest for the one-dimensional slab and increases for the cylinder and sphere. This behavior is to be expected due to the increased leakage with the curvi-linear geometries. The effect of increased leakage on the critical dimension can be also be seen for the forward peaked linear anisotropically scattering cases. For the reflected geometries, the critical dimension decreases with increasing reflector thickness. However, the critical dimension for the infinite lattice cell increases with the increasing moderator thickness. Even though this may seem counter-intuitive, it should be expected because the amount of interaction between the fissile medium and adjacent cells decreases with increasing moderator half thickness. ${ }^{34}$ 


\section{A. Isotropic Scattering}

1. Two-Group Pu-239.

Two-Group Isotropic Cross Sections

Tables 30 and 31 give the two-group, isotropic cross sections for $\mathrm{Pu}-239$.

Table 30: Fast Energy Group Macroscopic Cross Sections $\left(\mathrm{cm}^{-1}\right)$ for Pu-239

\begin{tabular}{|c|c|c|c|c|c|c|c|}
\hline Material & $\nu_{2}$ & $\Sigma_{2 f}$ & $\Sigma_{2 c}$ & $\Sigma_{22 s}$ & $\Sigma_{12 s}$ & $\Sigma_{2}$ & $\chi_{2}$ \\
\hline Pu-239 & 3.10 & 0.0936 & 0.00480 & 0.0792 & 0.0432 & 0.2208 & 0.575 \\
\hline
\end{tabular}

Table 31: Slow Energy Group Macroscopic Cross Sections $\left(\mathrm{cm}^{-1}\right)$ for Pu-239

\begin{tabular}{|c|c|c|c|c|c|c|c|}
\hline Material & $\nu_{1}$ & $\Sigma_{1 f}$ & $\Sigma_{1 c}$ & $\Sigma_{11 s}$ & $\Sigma_{21 s}$ & $\Sigma_{1}$ & $\chi_{1}$ \\
\hline Pu-239 & 2.93 & 0.08544 & 0.0144 & 0.23616 & 0.0 & 0.3360 & 0.425 \\
\hline
\end{tabular}

Infinite Medium (PU-2-0-IN)

Using the two-group isotropic Pu-239 cross-section set from Tables 30 and $31, \mathrm{k}_{\infty}=2.683767$ (problem 44) with a constant group angular and scalar flux and a group 2 to group 1 flux ratio $=0.675229$.

One-Medium Slab and Sphere Critical Dimensions

The critical dimensions, $\mathrm{r}_{c}$, are listed in Table 32 .

Table 32: Critical Dimensions, $r_{c}$, for Two-Group Bare Pu-239

\begin{tabular}{|c|c|c|c|c|c|}
\hline Problem & Identifier & Geometry & $\mathbf{r}_{c}(\mathrm{mfp})$ & $\mathbf{r}_{c}(\mathrm{~cm})$ & Reference \\
\hline 45 & PU-2-0-SL & Slab & 0.396469 & 1.795602 & $8,35,36$ \\
46 & PU-2-0-SP & Sphere & 1.15513 & 5.231567 & 8 \\
\hline
\end{tabular}




\section{Two-Group U-235.}

\section{Two-Group Cross Sections}

Tables 33 and 34 give the two-group, isotropic cross sections for U-235.

Table 33: Fast Energy Group Macroscopic Cross Sections $\left(\mathrm{cm}^{-1}\right)$ for U-235

\begin{tabular}{|c|c|c|c|c|c|c|c|}
\hline Material & $\nu_{2}$ & $\Sigma_{2 f}$ & $\Sigma_{2 c}$ & $\Sigma_{22 s}$ & $\Sigma_{12 s}$ & $\Sigma_{2}$ & $\chi_{2}$ \\
\hline U-235 & 2.70 & 0.06192 & 0.00384 & 0.078240 & 0.0720 & 0.2160 & 0.575 \\
\hline
\end{tabular}

Table 34: Slow Energy Group Macroscopic Cross Sections $\left(\mathrm{cm}^{-1}\right)$ for U-235

\begin{tabular}{|c|c|c|c|c|c|c|c|}
\hline Material & $\nu_{1}$ & $\Sigma_{1 f}$ & $\Sigma_{1 c}$ & $\Sigma_{11 s}$ & $\Sigma_{21 s}$ & $\Sigma_{1}$ & $\chi_{1}$ \\
\hline U-235 & 2.50 & 0.06912 & 0.01344 & 0.26304 & 0.0 & 0.3456 & 0.425 \\
\hline
\end{tabular}

Infinite Medium (U-2-0-IN)

Using the two-group U-235 cross-section set from Tables 33 and $34, \mathrm{k}_{\infty}=2.216349$ (problem 47) with a constant group angular and scalar flux and the group 2 to group 1 flux ratio $=0.474967$.

One-Medium Slab and Sphere Critical Dimensions

The critical dimensions, $\mathrm{r}_{c}$, are listed in Table 35 .

Table 35: Critical Dimension, $\mathrm{r}_{c}$, for Two-Group Bare U-235

\begin{tabular}{|c|c|c|c|c|c|}
\hline Problem & Identifier & Geometry & $\mathrm{r}_{c}(\mathrm{mfp})$ & $\mathbf{r}_{c}(\mathrm{~cm})$ & Reference \\
\hline 48 & U-2-0-SL & Slab & 0.649377 & 3.006375 & $8,35,36$ \\
49 & U-2-0-SP & Sphere & 1.70844 & 7.909444 & 8 \\
\hline
\end{tabular}




\section{Two-Group Uranium-Aluminum-Water Assembly.}

\section{Two-Group Isotropic Cross Sections}

Tables 36 and 37 gives the two-group, isotropic cross sections for the uranium, aluminum, and water assembly.

Table 36: Fast Energy Group Macroscopic Cross Sections $\left(\mathrm{cm}^{-1}\right)$ for U-Al

\begin{tabular}{|c|c|c|c|c|c|c|c|}
\hline Material & $\nu_{2}$ & $\Sigma_{2 f}$ & $\Sigma_{2 c}$ & $\Sigma_{22 s}$ & $\Sigma_{12 s}$ & $\Sigma_{2}$ & $\chi_{2}$ \\
\hline U-Al & 0.0 & 0.0 & 0.000222 & 0.247516 & 0.020432 & 0.26817 & 1.0 \\
\hline
\end{tabular}

Table 37: Slow Energy Group Macroscopic Cross Sections $\left(\mathrm{cm}^{-1}\right)$ for U-Al

\begin{tabular}{|c|c|c|c|c|c|c|c|}
\hline Material & $\nu_{1}$ & $\Sigma_{1 f}$ & $\Sigma_{1 c}$ & $\Sigma_{11 s}$ & $\Sigma_{21 s}$ & $\Sigma_{1}$ & $\chi_{1}$ \\
\hline U-Al & 2.83 & 0.06070636042 & 0.00314363958 & 1.21313 & 0.0 & 1.27698 & 0.0 \\
\hline
\end{tabular}

Infinite Medium (UAL-2-0-IN)

With the two-group cross-section set from Tables 36 and $37, \mathrm{k}_{\infty}=2.661745$ (problem 50) and the group 2 to group 1 flux ratio $=3.1250$.

One-Medium Slab and Sphere Critical Dimensions

Using the cross sections given in Tables 36 and 37 , the critical dimensions, $\mathrm{r}_{c}$, are given in Table 38 .

Table 38: Critical Dimensions, $\mathrm{r}_{c}$, for Two-Group Uranium-Aluminum-Water Assembly

\begin{tabular}{|c|c|c|c|c|c|}
\hline Problem & Identifier & Geometry & $\mathrm{r}_{c}(\mathrm{mfp})$ & $\mathrm{r}_{c}(\mathrm{~cm})$ & Reference \\
\hline 51 & UAL-2-0-SL & Slab & 2.09994 & 7.830630 & $8,35,36$ \\
52 & UAL-2-0-SP & Sphere & 4.73786 & 17.66738 & 8 \\
\hline
\end{tabular}




\section{Two-Group Uranium Research Reactor.}

Two-Group Isotropic Cross Sections

The cross sections for the one-medium (a), two-media (b and c), and infinite slab lattice (d) cases are different and are therefore listed separately. Tables 39 and 40 gives the two-group, one-medium, isotropic cross sections for the $93 \%$ enriched uranium bare university research reactor.

Table 39: Fast Energy Group Macroscopic Cross Sections $\left(\mathrm{cm}^{-1}\right)$ for Research Reactor (a)

\begin{tabular}{|c|c|c|c|c|c|c|c|}
\hline Material & $\nu_{2}$ & $\Sigma_{2 f}$ & $\Sigma_{2 c}$ & $\Sigma_{22 s}$ & $\Sigma_{12 s}$ & $\Sigma_{2}$ & $\chi_{2}$ \\
\hline Research Reactor (a) & 2.50 & 0.0010484 & 0.0010046 & 0.62568 & 0.029227 & 0.65696 & 1.0 \\
\hline
\end{tabular}

Table 40: Slow Energy Group Macroscopic Cross Sections $\left(\mathrm{cm}^{-1}\right)$ for Research Reactor (a)

\begin{tabular}{|c|c|c|c|c|c|c|c|}
\hline Material & $\nu_{1}$ & $\Sigma_{1 f}$ & $\Sigma_{1 c}$ & $\Sigma_{11 s}$ & $\Sigma_{21 s}$ & $\Sigma_{1}$ & $\chi_{1}$ \\
\hline Research Reactor (a) & 2.50 & 0.050632 & 0.025788 & 2.44383 & 0.0 & 2.52025 & 0.0 \\
\hline
\end{tabular}

Infinite Medium (URRa-2-0-IN)

The test set uses the two-group enriched U-235 cross-section set for the research reactor in Tables 39 and 40 with $\mathrm{k}_{\infty}=1.631452$ (problem 53) and the group 2 to group 1 flux ratio $=2.614706$.

One-Medium Slab and Sphere Critical Dimensions

The critical dimensions, $\mathrm{r}_{c}$, are listed in Table 41.

Table 41: Critical Dimensions, $\mathrm{r}_{c}$, for Two-Group Bare Research Reactor (a)

\begin{tabular}{|c|c|c|c|c|c|}
\hline Problem & Identifier & Geometry & $\mathrm{r}_{c}(\mathrm{mfp})$ & $\mathbf{r}_{c}(\mathrm{~cm})$ & Reference \\
\hline 54 & URRa-2-0-SL & Slab & 4.97112 & 7.566853 & $8,35,36$ \\
55 & URRa-2-0-SP & Sphere & 10.5441 & 16.049836 & 8 \\
\hline
\end{tabular}

\section{One-Medium Slab Scalar Neutron Fluxes}

Table 42 gives the normalized scalar neutron flux for the two-group bare research reactor (a) at four spatial points 36 . All values are normalized with the fast group flux at the center.

Table 42: Normalized Scalar Fluxes for Two-Group Bare Research Reactor (a)

\begin{tabular}{|c|c|c|c|c|c|c|c|}
\hline Problem & Identifier & Geometry & Energy Group & $\mathrm{r} / \mathrm{r}_{c}=0.241394$ & $\mathrm{r} / \mathrm{r}_{c}=\mathbf{0 . 5 0 2 9 0 5}$ & $\mathrm{r} / \mathrm{r}_{c}=\mathbf{0 . 7 4 4 3 0 0}$ & $\mathrm{r} / \mathrm{r}_{c}=1.0$ \\
\hline 54 & URRa-2-0-SL & Slab & Fast & 0.943363 & 0.761973 & 0.504012 & 0.147598 \\
& & & Slow & 0.340124 & 0.273056 & 0.173845 & $\mathbf{0 . 0 2 1 2 3 2 4}$ \\
\hline
\end{tabular}

Two-Media Cross Sections for Slab Geometry

The cross sections for the two-media problems for the uranium research reactor are given for two different multiplying media with one nonmultiplying reflector. The two multiplying materials are labeled (b) and $(c)$, 
respectively. The multiplying region consists of an $\mathrm{H}_{2} \mathrm{O}+\mathrm{U}-235$ mixture surrounded by a $\mathrm{H}_{2} \mathrm{O}$ reflector. The results in the literature for case $(c)$ only include the infinite water reflector. The cross sections are given in Tables 43 and 44 . Notice that this problem allows thermal upscattering in both multiplying and nonmultiplying regions.

Table 43: Fast Energy Group Macroscopic Cross Sections $\left(\mathrm{cm}^{-1}\right)$ for Research Reactor (b), (c) and $\mathrm{H}_{2} \mathrm{O}$ Reflector (a)

\begin{tabular}{|c|c|c|c|c|c|c|c|}
\hline Material & $\nu_{2}$ & $\Sigma_{2 f}$ & $\Sigma_{2 c}$ & $\Sigma_{22 s}$ & $\Sigma_{12 s}$ & $\Sigma_{2}$ & $\chi_{2}$ \\
\hline Research Reactor (b) & 2.50 & 0.000836 & 0.001104 & 0.83892 & 0.04635 & 0.88721 & 1.0 \\
Research Reactor (c) & 2.50 & 0.001648 & 0.001472 & 0.83807 & 0.04536 & 0.88655 & 1.0 \\
$\mathrm{H}_{2} \mathrm{O}$ (a) (ref) & 0.0 & 0.0 & 0.00074 & 0.83975 & 0.04749 & 0.88798 & 0.0 \\
\hline
\end{tabular}

Table 44: Slow Energy Group Macroscopic Cross Sections $\left(\mathrm{cm}^{-1}\right)$ for Research Reactor (b), (c) and $\mathrm{H}_{2} \mathrm{O}$ Reflector (a)

\begin{tabular}{|c|c|c|c|c|c|c|c|}
\hline Material & $\nu_{1}$ & $\Sigma_{1 f}$ & $\Sigma_{1 c}$ & $\Sigma_{11 s}$ & $\Sigma_{21 s}$ & $\Sigma_{1}$ & $\chi_{1}$ \\
\hline Research Reactor (b) & 2.50 & 0.029564 & 0.024069 & 2.9183 & 0.000767 & 2.9727 & 0.0 \\
Research Reactor (c) & 2.50 & 0.057296 & 0.029244 & 2.8751 & 0.00116 & 2.9628 & 0.0 \\
$\mathrm{H}_{2} \mathrm{O}$ (a) (refl) & 0.0 & 0.0 & 0.018564 & 2.9676 & 0.000336 & 2.9865 & 0.0 \\
\hline
\end{tabular}

Infinite Medium (URRb-2-0-IN and URRc-2-0-IN)

Using the two-group cross-section set for the research reactor $(b)$ from Tables 43 and $44, \mathrm{k}_{\infty}=1.365821$ (problem 56) with a constant group angular and scalar flux and the group 2 to group 1 flux ratio $=1.173679$. Using the two-group cross-ection set for research reactor (c) from Tables 43 and $44, \mathrm{k}_{\infty}=1.633380$ (problem 57) with a constant group angular and scalar flux and the group 2 to group 1 flux ratio $=1.933422$.

\section{Two-Media Slab Critical Dimensions}

Using the cross sections in Tables 43 and 44 for the $\mathrm{H}_{2} \mathrm{O}+\mathrm{U}-235$ research reactor and the $\mathrm{H}_{2} \mathrm{O}$ reflector, the critical dimensions are given in Table 45. The mfp results use the group 2 total macroscopic cross section of region $i$ to obtain the dimensions in $\mathrm{cm}$.

Table 45: Critical Dimensions for Two-Group Research Reactor (b),(c) with $\mathrm{H}_{2} \mathrm{O}$ Reflector (a)

\begin{tabular}{|c|c|c|c|c|c|c|}
\hline Problem & Identifier & Geometry & $\mathrm{U}-235, \mathrm{r}_{c}$ & $\mathrm{H}_{2} \mathrm{O}$ Width & $\mathrm{U}-235+\mathrm{H}_{2} \mathrm{O}$ Width & Ref. \\
\hline 58 & URRb-H2Oa(1)-2-0-SL & $\begin{array}{c}\text { Slab (mfp) } \\
(\mathrm{cm})\end{array}$ & $\begin{array}{c}5.94147 \\
6.696802\end{array}$ & $\begin{array}{c}\mathbf{1} \\
1.126152\end{array}$ & 7.822954 & 37 \\
\hline 59 & URRb-H2Oa(5)-2-0-SL & Slab (mfp) & 4.31485 & 5 & & 37 \\
\hline 60 & & $\begin{array}{c}(\mathrm{cm}) \\
\text { Slab }(\mathrm{mfp}) \\
(\mathrm{cm})\end{array}$ & $\begin{array}{c}4.863392 \\
4.15767 \\
4.686230\end{array}$ & $\begin{array}{c}5.630757 \\
\infty \\
\infty\end{array}$ & $\begin{array}{c}10.494149 \\
\infty \\
\infty\end{array}$ & 37 \\
\hline 61 & URRc-H2Oa(IN)-2-0-SL & $\begin{array}{c}\text { Slab (mfp) } \\
\text { (cm) }\end{array}$ & $\begin{array}{c}2.1826 \\
2.461903\end{array}$ & $\begin{array}{l}\infty \\
\infty\end{array}$ & $\begin{array}{l}\infty \\
\infty\end{array}$ & 37 \\
\hline
\end{tabular}


Two-Media Cross Sections for Infinite Slab Lattice Cell

The two-media cross sections are given in Tables 46 and 47 for a similar uranium enriched university research reactor. The $\nu_{2}$ is slightly unphysical to stress criticality codes. Two different reflector materials are also given in Tables 46 and 47 . The problems that use these cross sections are for an infinite slab lattice cell.

Table 46: Fast Group Macroscopic Cross Sections $\left(\mathrm{cm}^{-1}\right)$ for Research Reactor(d) and $\mathrm{H}_{2} \mathrm{O}$ Reflector (b), (c)

\begin{tabular}{|c|c|c|c|c|c|c|c|}
\hline Material & $\nu_{2}$ & $\Sigma_{2 f}$ & $\Sigma_{2 c}$ & $\Sigma_{22 s}$ & $\Sigma_{12 s}$ & $\Sigma_{2}$ & $\chi_{2}$ \\
\hline Research Reactor (d) & 1.004 & 0.61475 & 0.0019662 & 0.0 & 0.0342008 & 0.650917 & 1.0 \\
$\mathrm{H}_{2} \mathrm{O}$ (b) (ref) & 0.0 & 0.0 & $8.480293 \times 10^{-6}$ & 0.1096742149 & 0.001000595707 & 0.1106832906 & 0.0 \\
$\mathrm{H}_{2} \mathrm{O}$ (c) (ref) & 0.0 & 0.0 & $4.97229 \times 10^{-4}$ & 1.226381244 & 0.1046395340 & 1.331518007 & 0.0 \\
\hline
\end{tabular}

Table 47: Slow Group Macroscopic Cross Sections $\left(\mathrm{cm}^{-1}\right)$ for Research Reactor(d) and $\mathrm{H}_{2} \mathrm{O}$ Reflector (b), (c)

\begin{tabular}{|c|c|c|c|c|c|c|c|}
\hline Material & $\nu_{1}$ & $\Sigma_{1 f}$ & $\Sigma_{1 c}$ & $\Sigma_{11 s}$ & $\Sigma_{21 s}$ & $\Sigma_{1}$ & $\chi_{1}$ \\
\hline Research Reactor (d) & 2.50 & 0.045704 & 0.023496 & 2.06880 & 0.0 & 2.13800 & 0.0 \\
$\mathrm{H}_{2} \mathrm{O}$ (b) (refl) & 0.0 & 0.0 & 0.00016 & 0.36339 & 0.0 & 0.36355 & 0.0 \\
$\mathrm{H}_{2} \mathrm{O}$ (c) (refl) & 0.0 & 0.0 & 0.0188 & 4.35470 & 0.0 & 4.37350 & 0.0 \\
\hline
\end{tabular}

Infinite Medium (URRd-2-0-IN )

The test set uses the two-group cross-section set for the research reactor in Tables 46 and 47 with $\mathrm{k}_{\infty}=$ 1.034970 (problem 62) and the group 2 to group 1 flux ratio $=2.023344$.

Two-Media Infinite Slab Lattice Cell Critical Dimensions

Using the cross sections in Tables 46 and 47 for the enriched uranium research reactor with a $\mathrm{H}_{2} \mathrm{O}$ reflector, the critical dimensions for an infinite slab lattice cell as shown in Fig. 1 are given in Table 48. Because this is an infinite slab lattice cell with reflecting outer boundaries, notice that the moderator half thickness is given.

Table 48: Critical Dimensions for Two-Group Infinite Slab Lattice Cell and $\mathrm{H}_{2} \mathrm{O}$ Reflector (b), (c)

\begin{tabular}{|c|c|l|c|c|c|c|}
\hline Problem & \multicolumn{1}{|c|}{ Identifier } & Geometry & $\mathrm{U}-235, \mathrm{r}_{\mathrm{c}}$ & $\mathrm{H}_{2} \mathrm{O}$ Width & $\mathrm{U}-235+\mathrm{H}_{2} \mathrm{O}$ Width & Ref. \\
\hline 63 & URRd-H2Ob(1)-2-0-ISLC & Inf. Slab (mfp) & 0.02142 & 1 & & 34 \\
& & Lat. Cell (cm) & 0.0329074 & 9.034787 & 9.067695 & \\
64 & URRd-H2Ob(10)-2-0-ISLC & Inf. Slab (mfp) & 0.29951 & 10 & & 34 \\
& & Lat. Cell (cm) & 0.460135 & 90.347875 & 90.808010 & \\
\hline 65 & URRd-H2Oc(1)-2-0-ISLC & Inf. Slab (mfp) & 0.22197 & 1 & & 34 \\
& & Lat. Cell (cm) & 0.341011 & 0.751023 & 1.092034 & \multirow{3}{*}{64} \\
\cline { 3 - 7 } & \multirow{2}{*}{ URRd-H2Oc(10)-2-0-ISLC } & Inf. Slab (mfp) & 1.7699 & 10 & & \\
& & Lat. Cell (cm) & 2.719087 & 7.510225 & 10.229312 & \\
\hline
\end{tabular}




\section{Two-Group U- $\mathrm{D}_{2} \mathrm{O}$ Reactor.}

\section{Two-Group Isotropic Cross Sections}

Tables 49 and 50 give the two-group, isotropic cross sections for the uranium- $\mathrm{D}_{2} \mathrm{O}$ system.

Table 49: Fast Energy Group Macroscopic Cross Sections $\left(\mathrm{cm}^{-1}\right)$ for $\mathrm{U}^{-\mathrm{D}_{2} \mathrm{O}}$

\begin{tabular}{|c|c|c|c|c|c|c|c|}
\hline Material & $\nu_{2}$ & $\Sigma_{2 f}$ & $\Sigma_{2 c}$ & $\Sigma_{22 s}$ & $\Sigma_{12 s}$ & $\Sigma_{2}$ & $\chi_{2}$ \\
\hline $\mathrm{U}-\mathrm{D}_{2} \mathrm{O}$ & 2.50 & 0.002817 & 0.008708 & 0.31980 & 0.004555 & 0.33588 & 1.0 \\
\hline
\end{tabular}

Table 50: Slow Energy Group Macroscopic Cross Sections $\left(\mathrm{cm}^{-1}\right)$ for $\mathrm{U}_{2} \mathrm{D}$

\begin{tabular}{|c|c|c|c|c|c|c|c|}
\hline Material & $\nu_{1}$ & $\Sigma_{1 f}$ & $\Sigma_{1 c}$ & $\Sigma_{11 s}$ & $\Sigma_{21 s}$ & $\Sigma_{1}$ & $\chi_{1}$ \\
\hline $\mathrm{U}^{-} \mathrm{D}_{2} \mathrm{O}$ & 2.50 & $\mathbf{0 . 0 9 7}$ & 0.02518 & 0.42410 & 0.0 & 0.54628 & 0.0 \\
\hline
\end{tabular}

Infinite Medium (UD2O-2-0-IN )

The test set uses the two-group $\mathrm{U}-\mathrm{D}_{2} \mathrm{O}$ cross-section set from Tables 49 and 50 with $\mathrm{k}_{\infty}=1.000196$ (problem 67) and the group 2 to group 1 flux ratio $=26.823271$.

One-Medium Slab and Sphere Critical Dimensions

The critical dimensions, $\mathrm{r}_{c}$, are listed in Table 51.

Table 51: Critical Dimension, $\mathrm{r}_{c}$, for Two-Group $\mathrm{D}_{2} \mathrm{O}$ System

\begin{tabular}{|c|c|c|c|c|c|}
\hline Problem & Identifier & Geometry & $\mathrm{r}_{c}(\mathrm{mfp})$ & $\mathrm{r}_{c}(\mathrm{~cm})$ & Reference \\
\hline 68 & UD2O-2-0-SL & Slab & 284.367 & 846.632726 & $8,35,36$ \\
69 & UD2O-2-0-SP & Sphere & 569.430 & 1695.337621 & 8 \\
\hline
\end{tabular}




\section{B. Linearly Anisotropic Scattering}

The anisotropic scattering cross sections for the enriched U-235 research reactor and U-D $D_{2} \mathrm{O}$ reactor cases are the same as the isotropic set with the addition of the anisotropic cross sections, $\Sigma_{22 s_{1}}, \Sigma_{12 s_{1}}$, and $\Sigma_{11 s_{1}}$.

\section{Two-Group Uranium Research Reactor.}

Two-Group Anisotropic Macroscopic Cross Sections

Tables 52 and 53 gives the two-group, linearly anisotropic cross sections for the research reactor. Care must be used to correctly solve benchmark problem 71 because of the negative scattering for $\mu$ near $\mathbf{- 1}$.

Table 52: Fast Group Cross Sections for Linearly Anisotropic Scattering $\left(\mathrm{cm}^{-1}\right)$ Research Reactor (a)

\begin{tabular}{|c|c|c|c|c|c|c|c|c|c|}
\hline Material & $\nu_{2}$ & $\Sigma_{2 f}$ & $\Sigma_{2 c}$ & $\Sigma_{22 s_{0}}$ & $\Sigma_{22 s_{1}}$ & $\Sigma_{12 s_{0}}$ & $\Sigma_{12 s_{1}}$ & $\Sigma_{2}$ & $\chi_{2}$ \\
\hline Research Reactor (a) & 2.50 & 0.0010484 & 0.0010046 & 0.62568 & 0.27459 & 0.029227 & 0.0075737 & 0.65696 & 1.0 \\
\hline
\end{tabular}

Table 53: Slow Group Cross Sections for Linearly Anisotropic Scattering $\left(\mathrm{cm}^{-1}\right)$ Research Reactor (a)

\begin{tabular}{|c|c|c|c|c|c|c|c|c|}
\hline Material & $\nu_{1}$ & $\Sigma_{1 f}$ & $\Sigma_{1 c}$ & $\Sigma_{11 s_{0}}$ & $\Sigma_{11 s_{1}}$ & $\Sigma_{21 s}$ & $\Sigma_{1}$ & $\chi_{1}$ \\
\hline Research Reactor (a) & 2.50 & 0.050632 & 0.025788 & 2.44383 & 0.83318 & 0.0 & 2.52025 & 0.0 \\
\hline
\end{tabular}

Infinite Medium (URRa-2-1-IN )

The test set uses the two-group enriched U-235 cross-ection set from Tables 52 and 53 with $\mathrm{k}_{\infty}=1.631452$ (problem 70) and the group 2 to group 1 flux ratio $=2.614706$.

One-Medium Slab Critical Dimension

The critical dimensions are listed in Table 54.

Table 54: Critical Dimension, $\mathrm{r}_{c}$, for Two-Group Linearly Anisotropic Scattering Research Reactor (a)

\begin{tabular}{|c|c|c|c|c|c|}
\hline Problem & Identifier & Geometry & $\mathbf{r}_{c}(\mathrm{mfp})$ & $\mathbf{r}_{c}(\mathrm{~cm})$ & Reference \\
\hline 71 & URRa-2-1-SL & Slab & 6.2356 & 9.491600 & 25 \\
\hline
\end{tabular}

\section{One-Medium Slab Scalar Neutron Fluxes}

Table 55 gives the normalized scalar neutron flux for the two-group bare research reactor (a) with linearly anisotropic scattering at four spatial points. ${ }^{25}$ All values are normalized with the fast group flux at the center.

Table 55: Normalized Scalar Fluxes for Two-Group Bare Research Reactor (a)

\begin{tabular}{|c|c|c|c|c|c|c|c|}
\hline Problem & Identifier & Geometry & Energy Group & $\mathrm{r} / \mathrm{r}_{c}=0.20$ & $\mathrm{r} / \mathrm{r}_{c}=0.50$ & $\mathrm{r} / \mathrm{r}_{c}=0.80$ & $\mathrm{r} / \mathrm{r}_{c}=1.0$ \\
\hline 71 & URRa-2-1-SL & Slab & Fast & 0.963873 & 0.781389 & 0.472787 & 0.189578 \\
& & & Slow & 0.349006 & 0.280870 & 0.157376 & 0.0277639 \\
\hline
\end{tabular}




\section{Two-Group U-D $\mathrm{D}_{2} \mathrm{O}$ Reactor.}

Two-Group Anisotropic Macroscopic Cross Sections

Tables 56 and 57 gives the two-group, linearly anisotropic cross sections for the U- $\mathrm{D}_{2} \mathrm{O}$ system.

Table 56: Fast Energy Group Cross Sections for Linearly Anisotropic Scattering $\left(\mathrm{cm}^{-1}\right)$ for U- $\mathrm{D}_{2} \mathrm{O}$

\begin{tabular}{|c|c|c|c|c|c|c|c|c|c|}
\hline Material & $\nu_{2}$ & $\Sigma_{2 f}$ & $\Sigma_{2 c}$ & $\Sigma_{22 s_{0}}$ & $\Sigma_{22 s_{1}}$ & $\Sigma_{12 s_{0}}$ & $\Sigma_{12 s_{1}}$ & $\Sigma_{2}$ & $\chi_{2}$ \\
\hline $\mathrm{D}_{2} \mathrm{O}$ & 2.50 & 0.002817 & 0.008708 & 0.31980 & 0.06694 & 0.004555 & -0.0003972 & 0.33588 & 1.0 \\
\hline
\end{tabular}

Table 57: Slow Energy Group Cross Sections for Linearly Anisotropic Scattering $\left(\mathrm{cm}^{-1}\right)$ for U- $\mathrm{D}_{2} \mathrm{O}$

\begin{tabular}{|c|c|c|c|c|c|c|c|c|}
\hline Material & $\nu_{1}$ & $\Sigma_{1 f}$ & $\Sigma_{1 c}$ & $\Sigma_{11 s_{0}}$ & $\Sigma_{11 s_{1}}$ & $\Sigma_{21 s}$ & $\Sigma_{1}$ & $\chi_{1}$ \\
\hline $\mathrm{D}_{2} \mathrm{O}$ & 2.50 & 0.097 & 0.02518 & 0.42410 & 0.05439 & 0.0 & 0.54628 & 0.0 \\
\hline
\end{tabular}

Infinite Medium (UD2O-2-1-IN)

The test set uses the two-group linearly anisotropic $\mathrm{D}_{2} \mathrm{O}$ cross-section set from Tables 56 and 57 with $\mathbf{k}_{\infty}=1.000196$ (problem 72) and the group 2 to group 1 flux ratio $=26.823271$.

One-Medium Slab Critical Dimension

The critical dimension, $\mathbf{r}_{c}$, is listed in Table 58.

Table 58: Critical Dimension, $\mathrm{r}_{c}$, for Two-Group Linearly Anisotropic Scattering for $\mathrm{U}^{-\mathrm{D}_{2} \mathrm{O} \text { Reactor }}$

\begin{tabular}{|c|c|c|c|c|c|}
\hline Problem & Identifier & Geometry & $\mathbf{r}_{c}(\mathrm{mfp})$ & $\mathbf{r}_{c}(\mathrm{~cm})$ & Reference \\
\hline 73 & UD2O-2-1-SL & Slab & 336.05 & 1000.506133 & 25 \\
\hline
\end{tabular}




\section{THREE-ENERGY GROUP PROBLEM DEFINITIONS AND RESULTS}

A three-energy group isotropic infinite medium problem is defined in this section. The derivation appears in Appendix A. ${ }^{38,43}$ This problem assumes no thermal upscattering and no fission neutrons born in the slowest energy group.

The fast energy group is group 3 to be consistent with most of the references. Again, this notation is the reverse of most nuclear engineering textbooks.

The cross sections listed here are similar to the uranium university research reactors. Again, this problem uses cross sections that are reasonable representations of the materials described and are not general purpose values. The cross sections are intended to be used to verify algorithm performance and not to predict criticality experiments. The cross sections are from Ref. 38 and are derived in Appendix A.

Table 59: Fast Energy Group Macroscopic Cross Sections $\left(\mathrm{cm}^{-1}\right)$ for Research Reactor

\begin{tabular}{|c|c|c|c|c|c|c|c|c|}
\hline Material & $\nu_{3}$ & $\Sigma_{3 f}$ & $\Sigma_{3 c}$ & $\Sigma_{33 s}$ & $\Sigma_{23 s}$ & $\Sigma_{13 s}$ & $\Sigma_{3}$ & $\chi_{3}$ \\
\hline Research Reactor & 3.0 & 0.006 & 0.006 & 0.024 & 0.171 & 0.033 & 0.240 & 0.96 \\
\hline
\end{tabular}

Table 60: Middle Energy Group Macroscopic Cross Sections $\left(\mathrm{cm}^{-1}\right)$ for Research Reactor

\begin{tabular}{|c|c|c|c|c|c|c|c|c|}
\hline Material & $\nu_{2}$ & $\Sigma_{2 f}$ & $\Sigma_{2 c}$ & $\Sigma_{22 s}$ & $\Sigma_{32 s}$ & $\Sigma_{12 s}$ & $\Sigma_{2}$ & $\chi_{2}$ \\
\hline Research Reactor & 2.50 & 0.060 & 0.040 & 0.60 & 0.0 & 0.275 & 0.975 & 0.04 \\
\hline
\end{tabular}

Table 61: Slow Energy Group Macroscopic Cross Sections $\left(\mathrm{cm}^{-1}\right)$ for Research Reactor

\begin{tabular}{|c|c|c|c|c|c|c|c|c|}
\hline Material & $\nu_{1}$ & $\Sigma_{1 f}$ & $\Sigma_{1 c}$ & $\Sigma_{11 s}$ & $\Sigma_{21 s}$ & $\Sigma_{31 s}$ & $\Sigma_{1}$ & $\chi_{1}$ \\
\hline Research Reactor & 2.0 & 0.90 & 0.20 & 2.0 & 0.0 & 0.0 & 3.10 & 0.0 \\
\hline
\end{tabular}

Infinite Medium (URR-3-0-IN)

Using the three-group cross-section set from Tables 59,60 , and $61, \mathrm{k}_{\infty}=1.60$ (problem 74) with a constant group angular and scalar flux and the group 2 to group 3 flux ratio $=0.480$, the group 1 to group 2 flux ratio $=0.3125$, and the group 1 to group 3 flux ratio $=0.150$. 


\section{AND RESULTS}

A six-energy group isotropic infinite medium problem comprised of two coupled three-energy group cross sections used in URR-3-0-IN is defined in this section. This test problem defines a six group cross-section set $^{39}$. such that energy groups 6 and 1, 5 and 2 , and 4 and 3 are equivalent. The top three groups are decoupled from the lower three groups except for the fission distribution, $\chi_{i}$, which affects energy groups 6 , 5, 2, and 1. Energy group 6 (group 1) scatters to groups 5 and 4 (groups 2 and 3). Energy group 5 (group 2) scatters to group 4 (group 3). Energy group 4 (group 3) self-scatters only. Since groups 1,2, and 3 are upscatter equivalents of groups 6,5 , and 4 , respectively, this problem should only be used with codes that allow for thermal upscattering.

Table 62: Fast Energy Group 6 Macroscopic Cross Sections $\left(\mathrm{cm}^{-1}\right)$ for Research Reactor

\begin{tabular}{|c|c|c|c|c|c|c|c|c|}
\hline Material & $\nu_{6}$ & $\Sigma_{6 f}$ & $\Sigma_{6 c}$ & $\Sigma_{66 s}$ & $\Sigma_{56 s}$ & $\Sigma_{46 s}$ & $\Sigma_{6}$ & $\chi_{6}$ \\
\hline Research Reactor & 3.0 & 0.006 & 0.006 & 0.024 & 0.171 & 0.033 & 0.240 & 0.48 \\
\hline
\end{tabular}

Table 63: Energy Group 5 Macroscopic Cross Sections $\left(\mathrm{cm}^{-1}\right)$ for Research Reactor

\begin{tabular}{|c|c|c|c|c|c|c|c|c|}
\hline Material & $\nu_{5}$ & $\Sigma_{5 f}$ & $\Sigma_{5 c}$ & $\Sigma_{55 s}$ & $\Sigma_{65 s}$ & $\Sigma_{45 s}$ & $\Sigma_{5}$ & $\chi_{5}$ \\
\hline Research Reactor & 2.50 & 0.060 & 0.040 & 0.60 & 0.0 & 0.275 & 0.975 & 0.02 \\
\hline
\end{tabular}

Table 64: Energy Group 4 Macroscopic Cross Sections $\left(\mathrm{cm}^{-1}\right)$ for Research Reactor

\begin{tabular}{|c|c|c|c|c|c|c|c|c|}
\hline Material & $\nu_{4}$ & $\Sigma_{4 f}$ & $\Sigma_{4 c}$ & $\Sigma_{44 s}$ & $\Sigma_{54 s}$ & $\Sigma_{64 s}$ & $\Sigma_{4}$ & $\chi_{4}$ \\
\hline Research Reactor & 2.0 & 0.90 & 0.20 & 2.0 & 0.0 & 0.0 & 3.10 & 0.0 \\
\hline
\end{tabular}

Table 65: Energy Group 3 Macroscopic Cross Sections $\left(\mathrm{cm}^{-1}\right)$ for Research Reactor

\begin{tabular}{|c|c|c|c|c|c|c|c|c|}
\hline Material & $\nu_{3}$ & $\Sigma_{3 f}$ & $\Sigma_{3 c}$ & $\Sigma_{33 s}$ & $\Sigma_{23 s}$ & $\Sigma_{13 s}$ & $\Sigma_{3}$ & $\chi_{3}$ \\
\hline Research Reactor & 2.0 & 0.90 & 0.20 & 2.0 & 0.0 & 0.0 & 3.10 & 0.0 \\
\hline
\end{tabular}

Table 66: Energy Group 2 Macroscopic Cross Sections $\left(\mathrm{cm}^{-1}\right)$ for Research Reactor

\begin{tabular}{|c|c|c|c|c|c|c|c|c|}
\hline Material & $\nu_{2}$ & $\Sigma_{2 f}$ & $\Sigma_{2 c}$ & $\Sigma_{22 s}$ & $\Sigma_{12 s}$ & $\Sigma_{32 s}$ & $\Sigma_{2}$ & $\chi_{2}$ \\
\hline Research Reactor & 2.50 & 0.060 & 0.040 & 0.60 & 0.0 & 0.275 & 0.975 & 0.02 \\
\hline
\end{tabular}

Table 67: Slow Energy Group 1 Macroscopic Cross Sections $\left(\mathrm{cm}^{-1}\right)$ for Research Reactor

\begin{tabular}{|c|c|c|c|c|c|c|c|c|}
\hline Material & $\nu_{1}$ & $\Sigma_{1 f}$ & $\Sigma_{1 c}$ & $\Sigma_{11 s}$ & $\Sigma_{21 s}$ & $\Sigma_{31 s}$ & $\Sigma_{1}$ & $\chi_{1}$ \\
\hline Research Reactor & 3.0 & 0.006 & 0.006 & 0.024 & 0.171 & 0.033 & 0.240 & 0.48 \\
\hline
\end{tabular}


Infinite Medium (URR-6-0-IN)

Since this problem is comprised of problem URR-3-0-IN cross sections with modified $\chi_{i}$ values, the final $\mathrm{k}_{\infty}$ value and flux ratios will not change. Using the six-group cross-section set from Tables $62,63,6465$, 66 , and $67, \mathrm{k}_{\infty}=1.60$ (problem 75) with a constant angular and scalar flux in each group. The group 5 to group 6 and group 2 to group 1 flux ratio $=0.480$, the group 4 to group 5 and group 3 to group 2 flux ratio $=0.3125$, and a group 4 to group 6 and group 3 to group 1 flux ratio $=0.150$. These ratios are the same as in the three-group problem. 


\section{SUMMARY}

In this report, we have documented 75 problem descriptions with precise results for the critical dimensions, $\mathbf{k}_{\text {eff }}$ eigenvalue, and some eigenfunction (scalar neutron flux) results for infinite, slab, cylindrical, and spherical geometries for one- and two-energy group, multiple-media, and both isotropic and linearly anisotropic scattering using the listed references. We have not given a complete listing of every referenced result that has been published. Instead, we have included the references that provide both true transport solutions and enough information to reproduce the results. Several other references are included for reference completeness. All test set problems specifications and results are from peer reviewed journals, and have, in many cases, been solved numerically by more than one analytic method. These calculated values for $\mathrm{k}_{\text {eff }}$ and the scalar neutron flux are believed to be accurate to at least five decimal places. Criticality codes can be verified using these analytic benchmark test problems.

\section{ACKNOWLEDGMENTS}

The authors would like to thank the following people for their role in making this document as correct and useful as possible: R.E. Alcouffe, R.A. Axford (U of llinois), G.E. Bosler, F.B. Brown, J.D. Court, B.D. Ganapol (U of Arizona), R.P. Gardner (NCSU), D.E. Kornreich, E.W. Larsen (U of Michigan), R.C. Little, R.D. O'Dell, L.M. Petrie (ORNL), A.K. Prinja (UNM), J.E. Stewart, and P.P. Whalen. R.D. O'Dell provided the three- and six-group infinite medium problems. 


\section{REFERENCES}

[1] "IEEE Software Engineering Standards Collection - Spring 1991 Edition" IEEE, Inc (1991).

[2] Argonne National Laboratory "Argonne Code Center: Benchmark Problem Book" Mathematics and Computation Division of the American Nuclear Society, ANL-7416 (1968).

[3] Briggs, J.B. Criticality Safety Benchmark Specification Handbook NEA/NSC/DOC(95)03/I Volume I.

[4] Petrie, L.M. LA-12556-C, Proceedings of the First Annual Nuclear Criticality Safety Technology Project. 18 (1992).

[5] Lee, C.E. and Carruthers, L.M. "Comparison of $\mathrm{S}_{N}$ Quadrature Methods in Benchmark Criticality Calculations" LA-9751-MS UC-82 (1983).

[6] Case, K.M. and Zweifel, P.F. "Linear Transport Theory," Addison-Wesley Publ. Co. , Reading, Mass. (1967).

[7] Grandjean, P. and Siewert, C.E. "The $\mathrm{F}_{N}$ Method in Neutron Transport Theory. Part II: Applications and Numerical Results" Nucl. Sci. Eng., 69, 161 (1979).

[8] Siewert, C.E. and Thomas, J.R. "On Two-Group Critical Problems in Neutron Transport Theory" Nucl. Sci. Eng. 94, 264 (1986).

[9] Kornreich, D.E., and Ganapol, B.D. "The Green's Function Method For Nuclear Engineering Applications" Nucl. Sci. Eng., 126, 293 (1997).

[10] Shuttleworth, T. "The Verification of Monte Carlo Codes in Middle Earth" Proceedings of 8th International Conference on Radiation Shielding, p 1148 (1994).

[11] Gelbard, E.M. and Prael, R.E. "Computation of Standard Deviations in Eigenvalue Calculations" Prog. Nucl. Energy, 24, 237 (1990).

[12] Duderstadt, J.J, and Hamilton, L.J. "Nuclear Reactor Analysis," John Wiley and Sons, NY (1976).

[13] Atalay, M.A. "The Reflected Slab and Sphere Criticality Problem with Anisotropic Scattering in OneSpeed Neutron Transport Theory" Prog. Nucl. Energy, 31, 299 (1997).

[14] Atalay, M.A. "The Critical Slab Problem for Reflecting Boundary Conditions in One-Speed Neutron Transport Theory" Ann. Nucl. Energy, 23, 183 (1996).

[15] Dahl, E.B. and Sjostrand, N.G. "Eigenvalue Spectrum of Multiplying Slabs and Spheres for Monoenergetic Neutrons with Anisotropic Scattering" Nucl. Sci. Eng., 69, 114 (1979).

[16] Sahni, D.C. Dahl, E.B., and Sjostrand, N.G. "Real Criticality Eigenvalues of One-Speed Linear Transport Operator" Trans. Th. Stat. Phy, 24, 1295 (1995).

[17] Garis, N.S. "One-Speed Neutron Transport Eigenvalues for Reflected Slabs and Spheres" Nucl. Sci. Eng., 107, 343 (1991).

[18] Sahni, D.C. and Sjostrand, N.G. "Critical and Time Eigenvalues in One-Speed Neutron Transport" Prog. Nucl. Energy, 23, 3, 241 (1990).

[19] Sahni, D.C. "Some New Results Pertaining to Criticality and Time Eigenvalues of One-Speed Neutron Transport Equation" Prog. Nucl. Energy, 30, 3, 305 (1996).

[20] Sjostrand, N.G. "Criticality of Reflected Spherical Reactors For Neutrons of One-Speed" Prog. Nucl. Energy, 13, 533 (1986).

[21] Bell, G.I. and Glasstone, S. "Nuclear Reactor Theory," Van Nostrand Reinhold Co. (1970). 
[22] Burkart, A.R., Ishiguro, Y., and Siewert, C.E. "Neutron Transport in Two Dissimilar Media with Anisotropic Scattering," Nucl. Sci. Eng., 61, 72 (1976).

[23] Burkart, A. R. "The Application of Invariance Principles to Critical Problems in Reflected Reactors" PhD. Thesis North Carolina State University (1975).

[24] Boff, V.C., Molinari, V.G. and Spiga, G. "Anisotropy of Scattering and Fission in Neutron Transport Theory," Nucl. Sci. Eng., 64, 823 (1977).

[25] Bosler, G.E. "Critical Slab Solution to the Two-Group Neutron Transport Equation for Linearly Anisotropic Scattering" PhD. Thesis University of Virginia (1972).

[26] Kaper, H.G., Lindeman, A.J., and Leaf, G.K. "Benchmark Values for the Slab and Sphere Criticality Problem in One-Group Neutron Transport Theory" Nucl. Sci. Eng., 54, 94 (1974).

[27] Westfall, R.M. "Benchmark Solutions for the Infinite Critical Cylinder" Trans. Am. Nucl. Soc., 44, 281 (1983).

[28] Westfall, R.M. and Metcalf, D.R. "Exact Solution of the Transport Equation for Critical Cylindrical Configurations" Trans. Am. Nucl. Soc., 15, 266 (1972).

[29] Westfall, R.M. and Metcalf, D.R. "Singular Eigenfunction Solution of the Monoenergetic Neutron Transport Equation for Finite Radially Reflected Critical Cylinders" Nucl. Sci. Eng., 52, 1 (1973).

[30] Lathrop, K.D. and Leonard, A. "Comparisons of Exact and $S_{N}$ Solutions of the Monoenergetic Critical Equation with Anisotropic Scattering" Nucl. Sci. Eng., 22, 115 (1965).

[31] Sanchez, R. and Ganapol, B.D. "Benchmark Values for Monoenergetic Neutron Transport in OneDimensional Cylindrical Geometry with Linearly Anisotropic Scattering" Nucl. Sci. Eng., 64, 61 (1983).

[32] Argonne National Laboratory "Reactor Physics Constants" United States Atomic Energy Commission, ANL-5800 (1963).

[33] Stewart, J.E. and Metcalf, D.R. "Monoenergetic Neutron Transport in a Finite Critical Reflected Slab System" Trans. Am. Nucl. Soc., 15, 267 (1972).

[34] Stewart, J.E. "Two-Media Solutions to the Neutron Transport Equation" PhD. Thesis University of Virginia (1974).

[35] Forster, R.A. and Metcalf, D.R. "Two-Group Transport Solutions to the One-Dimensional Critical Slab Problem" Trans. Am. Nucl. Soc., 12, 637 (1969).

[36] Forster, R.A. "Two-Group Critical Slab Problem" PhD. Thesis University of Virginia, (1970).

[37] Ishiguro,Y. and Garcia, R.D.M. "Two-Media Problems in Two-Group Neutron Transport Theory" Nucl. Sci. Eng., 68, 99 (1978).

[38] O'Dell, R.D. “QA Problem-3 Group $k_{\infty}$ Calculation" Private Communication (1998).

[39] O'Dell, R.D. "QA Test 2: Upscatter Convergence" Private Communication (1998).

[40] Henry, A.F. "Nuclear-Reactor Analysis" MIT Press, Cambridge, MA, (1975).

[41] Lewis, E.E. and Miller, W.F. "Computational Methods in Neutron Transport," American Nuclear Society, Inc, La Grange Park, Il (1993).

[42] Whalen, P.P. Private Communication (1998).

[43] Parsons, D.K. and Nigg, D.W. "Extension of the Analytic Nodal Method for Four Energy Groups" Trans. Am. Nuc. Soc. 50, 282 (1985).

[44] Larsen, E.W. Private Communication (1998).

[45] Kornreich, D.E., Private Communication (1998). 


\section{APPENDIX A \\ Derivation of One-, Two-, and Three-Group $\mathbf{k}_{\infty}$}

To follow the benchmark referenced literature for the multi-group problems, the lowest energy group is group 1. This notation is the reverse from most nuclear engineering textbooks.

\section{One-Energy Group Infinite Medium $\mathbf{k}_{\infty}$}

For an infinite, isotropic, homogeneous medium, the neutron leakage term, $\Omega \cdot \nabla \Psi=0$, and the angular and scalar neutron flux is constant everywhere. Integrating the one-energy group infinite medium form of the transport equation over angle produces:

$$
\Sigma_{t} \phi=\Sigma_{s} \phi+\frac{\nu \Sigma_{f}}{\mathrm{k}_{\infty}} \phi
$$

where $\phi$ is the scalar neutron flux. The equation can be directly solved for $k_{\infty}$.

$$
\mathrm{k}_{\infty}=\frac{\nu \Sigma_{f}}{\Sigma_{t}-\Sigma_{s}}
$$

or, in terms of mean number of secondaries, $c$ :

$$
\mathrm{k}_{\infty}=c\left[\frac{\nu \Sigma_{f} \Sigma_{t}}{\left(\Sigma_{t}-\Sigma_{s}\right)\left(\Sigma_{s}+\nu \Sigma_{f}\right)}\right]
$$

\section{Two-Energy Group Infinite Medium $\mathbf{k}_{\infty}$}

The two-group infinite medium form of the neutron transport equation reduces to:

$$
\begin{aligned}
& \Sigma_{2} \phi_{2}=\Sigma_{22 s} \phi_{2}+\Sigma_{21 s} \phi_{1}+\frac{\chi_{2}}{k_{\infty}}\left[\nu_{2} \Sigma_{2 f} \phi_{2}+\nu_{1} \Sigma_{1 f} \phi_{1}\right] \\
& \Sigma_{1} \phi_{1}=\Sigma_{11 s} \phi_{1}+\Sigma_{12 s} \phi_{2}+\frac{\chi_{1}}{k_{\infty}}\left[\nu_{1} \Sigma_{1 f} \phi_{1}+\nu_{2} \Sigma_{2 f} \phi_{2}\right]
\end{aligned}
$$

Rearranging the equations in terms of $\phi_{1}$ and $\phi_{2}$ :

$$
\begin{aligned}
& {\left[\Sigma_{2}-\Sigma_{22 s}-\frac{\chi_{2}}{k_{\infty}} \nu_{2} \Sigma_{2 f}\right] \phi_{2}-\left[\Sigma_{21 s}+\frac{\chi_{2}}{k_{\infty}} \nu_{1} \Sigma_{1 f}\right] \phi_{1}=0} \\
& {\left[\Sigma_{1}-\Sigma_{11 s}-\frac{\chi_{1}}{k_{\infty}} \nu_{1} \Sigma_{1 f}\right] \phi_{1}-\left[\Sigma_{12 s}+\frac{\chi_{1}}{k_{\infty}} \nu_{2} \Sigma_{2 f}\right] \phi_{2}=0}
\end{aligned}
$$


This equation can be written in matrix form as: ${ }^{42}$

$$
\left[\begin{array}{cc}
-\left(\Sigma_{21 s}+\frac{\chi_{2}}{k_{\infty}} \nu_{1} \Sigma_{1 f}\right) & \left(\Sigma_{2}-\Sigma_{22 s}-\frac{\chi_{2}}{k_{\infty}} \nu_{2} \Sigma_{2 f}\right) \\
\left(\Sigma_{1}-\Sigma_{11 s}-\frac{\chi_{1}}{k_{\infty}} \nu_{1} \Sigma_{1 f}\right) & -\left(\Sigma_{12 s}+\frac{\chi_{1}}{k_{\infty}} \nu_{2} \Sigma_{2 f}\right)
\end{array}\right]\left[\begin{array}{l}
\phi_{1} \\
\phi_{2}
\end{array}\right]=\left[\begin{array}{l}
0 \\
0
\end{array}\right]
$$

To simplify the matrix elements, it is useful to define a total removal cross section, $\Sigma_{g}$, for each energy group $g$ as the difference between the total cross section and in-group scattering or: ${ }^{40}$

$$
\begin{aligned}
& \Sigma_{2}^{r e m}=\Sigma_{2}-\Sigma_{22 s} \\
& \Sigma_{1}^{r e m}=\Sigma_{1}-\Sigma_{11 s}
\end{aligned}
$$

Setting the determinant of this matrix equal to zero will give an equation that can be solved for $k_{\infty}$. One solution is $\mathrm{k}_{\infty}=0$. The other solution is:

$$
k_{\infty}=\frac{\chi_{1}\left(\nu_{2} \Sigma_{2 f} \Sigma_{21 s}+\Sigma_{2}^{r e m} \nu_{1} \Sigma_{1 f}\right)+\chi_{2}\left(\nu_{1} \Sigma_{1 f} \Sigma_{12 s}+\Sigma_{1}^{r e m} \nu_{2} \Sigma_{2 f}\right)}{\Sigma_{1}^{r e m} \Sigma_{2}^{r e m}-\Sigma_{12 s} \Sigma_{21 s}}
$$

If there is no thermal upscattering, the equation reduces to:

$$
k_{\infty}=\frac{\chi_{1} \nu_{1} \Sigma_{1 f}}{\Sigma_{1}^{r e m}}+\chi_{2}\left(\frac{\nu_{1} \Sigma_{1 f} \Sigma_{12 s}}{\Sigma_{1}^{r e m} \Sigma_{2}^{r e m}}+\frac{\nu_{2} \Sigma_{2 f}}{\Sigma_{2}^{r e m}}\right)
$$

To obtain the flux ratio, Eqs. 23 and 24 are added to eliminate $\chi_{1}$ and $\chi_{2}$ to give:

$$
\left[\Sigma_{1}^{r e m}-\Sigma_{21 s}-\frac{\nu_{1} \Sigma_{1 f}}{k_{\infty}}\right] \phi_{1}+\left[\Sigma_{2}^{r e m}-\Sigma_{12 s}-\frac{\nu_{2} \Sigma_{2 f}}{k_{\infty}}\right] \phi_{2}=0
$$

where $\chi_{1}+\chi_{2}=1$.

Solving for $\phi_{2} / \phi_{1}$ :

$$
\frac{\phi_{2}}{\phi_{1}}=\frac{\left[\Sigma_{1}^{r e m}-\Sigma_{21 s}-\frac{\nu_{1} \Sigma_{3 f}}{k_{\infty}}\right]}{\left[\frac{\nu_{2} \Sigma_{2 f}}{k_{\infty}}-\Sigma_{2}^{r e m}+\Sigma_{12 s}\right]}
$$

If there is no thermal upscattering, the equation reduces to:

$$
\frac{\phi_{2}}{\phi_{1}}=\frac{\left[\Sigma_{1}^{r e m}-\frac{\nu_{1} \Sigma_{1 f}}{k_{\infty}}\right]}{\left[\frac{\nu_{2} \Sigma_{2 f}}{k_{\infty}}-\Sigma_{2}^{r e m}+\Sigma_{12 s}\right]}
$$




\section{Three-Energy Group Infinite Medium $\mathbf{k}_{\infty}(\mathbf{a})$}

To make the three-energy group problem simpler, the following assumptions are made: ${ }^{40}$

- No thermal upscattering from group $\mathrm{j}$ to group $\mathrm{i}, j<i, \Sigma_{i j_{s}}=0$

- No fission neutrons are born in the lowest energy group, i.e., $\chi_{1}=0$

The neutron transport equation can be written as:

$$
\begin{aligned}
& \Sigma_{3} \phi_{3}=\Sigma_{33 s} \phi_{3}+\frac{\chi_{3}}{k_{\infty}}\left[\nu_{3} \Sigma_{3 f} \phi_{3}+\nu_{2} \Sigma_{2 f} \phi_{2}+\nu_{1} \Sigma_{1 f} \phi_{1}\right] \\
& \Sigma_{2} \phi_{2}=\Sigma_{22 s} \phi_{2}+\Sigma_{23 s} \phi_{3}+\frac{\chi_{2}}{k_{\infty}}\left[\nu_{3} \Sigma_{3 f} \phi_{3}+\nu_{2} \Sigma_{2 f} \phi_{2}+\nu_{1} \Sigma_{1 f} \phi_{1}\right] \\
& \Sigma_{1} \phi_{1}=\Sigma_{11 s} \phi_{1}+\Sigma_{12 s} \phi_{2}+\Sigma_{13 s} \phi_{2}
\end{aligned}
$$

Rearranging the equations in terms of $\phi_{1}, \phi_{2}$ and $\phi_{3}$ :

$$
\begin{aligned}
{\left[\Sigma_{3}-\Sigma_{33 s}-\frac{\chi_{3}}{k_{\infty}} \nu_{3} \Sigma_{3 f}\right] \phi_{3}-\left[\frac{\chi_{3}}{k_{\infty}} \nu_{2} \Sigma_{2 f}\right] \phi_{2}-\left[\frac{\chi_{3}}{k_{\infty}} \nu_{1} \Sigma_{1 f}\right] \phi_{1} } & =0 \\
-\left[\Sigma_{23 s}+\frac{\chi_{2}}{k_{\infty}} \nu_{3} \Sigma_{3 f}\right] \phi_{3}+\left[\Sigma_{2}-\Sigma_{22 s}-\frac{\chi_{2}}{k_{\infty}} \nu_{2} \Sigma_{2 f}\right] \phi_{2}-\left[\frac{\chi_{2}}{k_{\infty}} \nu_{1} \Sigma_{1 f}\right] \phi_{1} & =0 \\
-\Sigma_{13 s} \phi_{3}-\Sigma_{12 s} \phi_{2}+\left[\Sigma_{1}-\Sigma_{11 s}\right] \phi_{1} & =0
\end{aligned}
$$

This equation can be written in matrix form as: ${ }^{40}$

$$
\left[\begin{array}{ccc}
\left(\Sigma_{3}-\Sigma_{33 s}-\frac{\chi_{3}}{k_{\infty}} \nu_{3} \Sigma_{3 f}\right) & -\left(\frac{\chi_{3}}{k_{\infty}} \nu_{2} \Sigma_{2 f}\right) & -\left(\frac{\chi_{3}}{k_{\infty}} \nu_{1} \Sigma_{1 f}\right) \\
-\left(\Sigma_{23 s}+\frac{\chi_{2}}{k_{\infty}} \nu_{3} \Sigma_{3 f}\right) & \left(\Sigma_{2}-\Sigma_{22 s}-\frac{\chi_{2}}{k_{\infty}} \nu_{2} \Sigma_{2 f}\right) & -\left(\frac{\chi_{2}}{k_{\infty}} \nu_{1} \Sigma_{1 f}\right) \\
-\Sigma_{13 s} & -\Sigma_{12 s} & \left(\Sigma_{1}-\Sigma_{11 s}\right)
\end{array}\right]\left[\begin{array}{l}
\phi_{1} \\
\phi_{2} \\
\phi_{3}
\end{array}\right]=\left[\begin{array}{l}
0 \\
0 \\
0
\end{array}\right]
$$

Using the total removal cross sections defined in Eqs. 26 and 27, the determinant of the matrix then becomes:

$$
\left[\begin{array}{ccc}
\left(\Sigma_{3}^{r e m}-\frac{\chi_{3}}{k_{\infty}} \nu_{3} \Sigma_{3 f}\right) & -\left(\frac{\chi_{3}}{k_{\infty}} \nu_{2} \Sigma_{2 f}\right) & -\left(\frac{\chi_{3}}{k_{\infty}} \nu_{1} \Sigma_{1 f}\right) \\
-\left(\Sigma_{23 s}+\frac{\chi_{2}}{k_{\infty}} \nu_{3} \Sigma_{3 f}\right) & \left(\Sigma_{2}^{r e m}-\frac{\chi_{2}}{k_{\infty}} \nu_{2} \Sigma_{2 f}\right) & -\left(\frac{\chi_{2}}{k_{\infty}} \nu_{1} \Sigma_{1 f}\right) \\
-\Sigma_{13 s} & -\Sigma_{12 s} & \Sigma_{1}^{r e m}
\end{array}\right]
$$

If we multiply the second line by $\chi_{3}$, multiply the first line by $\chi_{2}$, and subtract the results, and multiply the first line by $\mathrm{k}_{\infty}$, the determinant becomes: 


$$
\left[\begin{array}{ccc}
\left(\Sigma_{3}^{r e m} k_{\infty}-\chi_{3} \nu_{3} \Sigma_{3 f}\right) & -\left(\chi_{3} \nu_{2} \Sigma_{2 f}\right) & -\left(\chi_{3} \nu_{1} \Sigma_{1 f}\right) \\
-\left(\chi_{3} \Sigma_{23 s}+\chi_{2} \Sigma_{3}^{r e m}\right) & \left(\chi_{3} \Sigma_{2}^{r e m}\right) & 0 \\
-\Sigma_{13 s} & -\Sigma_{12 s} & \Sigma_{1}^{r e m}
\end{array}\right]
$$

Two of the $\mathbf{k}_{\infty}$ solutions are zero. The other solution is:

$$
k_{\infty}=\frac{\left(\chi_{3} \Sigma_{23 s}+\chi_{2} \Sigma_{3}^{r e m}\right)\left(\nu_{1} \Sigma_{1 f} \Sigma_{12 s}+\nu_{2} \Sigma_{2 f} \Sigma_{1}^{r e m}\right)+\chi_{3} \Sigma_{2}^{r e m}\left(\nu_{1} \Sigma_{1 f} \Sigma_{13 s}+\nu_{3} \Sigma_{3 f} \Sigma_{1}^{r e m}\right)}{\Sigma_{1}^{r e m} \Sigma_{2}^{r e m} \Sigma_{3}^{r e m}}
$$

\section{Three-Energy Group Infinite Medium $\mathbf{k}_{\infty}(\mathbf{b})$}

An alternative method for solving the three-group $k_{\infty}$ problem $^{38}$ is to rearrange the three-group transport equations in Eqs. 33, 34, 35:

$$
\begin{aligned}
& \left(\Sigma_{3}-\Sigma_{33 s}\right) \phi_{3}=\frac{\chi_{3}}{k_{\infty}}\left[\nu_{3} \Sigma_{3 f} \phi_{3}+\nu_{2} \Sigma_{2 f} \phi_{2}+\nu_{1} \Sigma_{1 f} \phi_{1}\right] \\
& \left(\Sigma_{2}-\Sigma_{22 s}\right) \phi_{2}=\Sigma_{23 s} \phi_{3}+\frac{\chi_{2}}{k_{\infty}}\left[\nu_{3} \Sigma_{3 f} \phi_{3}+\nu_{2} \Sigma_{2 f} \phi_{2}+\nu_{1} \Sigma_{1 f} \phi_{1}\right] \\
& \left(\Sigma_{1}-\Sigma_{11 s}\right) \phi_{1}=\Sigma_{12 s} \phi_{2}+\Sigma_{13 s} \phi_{3}
\end{aligned}
$$

Divide each equation by $\phi_{3}$ and define:

$$
\begin{aligned}
\phi_{23} & =\frac{\phi_{2}}{\phi_{3}} \\
\phi_{13} & =\frac{\phi_{1}}{\phi_{3}} \\
\Sigma_{i}^{r e m} & =\Sigma_{i}-\Sigma_{i i s}
\end{aligned}
$$

The result gives:

$$
\begin{aligned}
\Sigma_{3}^{r e m} & =\frac{\chi_{3}}{k_{\infty}}\left[\nu_{3} \Sigma_{3 f}+\nu_{2} \Sigma_{2 f} \phi_{23}+\nu_{1} \Sigma_{1 f} \phi_{13}\right] \\
\phi_{23} \Sigma_{2}^{r e m} & =\Sigma_{23 s}+\frac{\chi_{2}}{k_{\infty}}\left[\nu_{3} \Sigma_{3 f}+\nu_{2} \Sigma_{2 f} \phi_{23}+\nu_{1} \Sigma_{1 f} \phi_{13}\right] \\
\phi_{13} \Sigma_{1}^{r e m} & =\Sigma_{13 s}+\Sigma_{12 s} \phi_{23}
\end{aligned}
$$


If we divide Eqs. 50 and 51 by $\nu_{3} \Sigma_{3 f}$ and define:

$$
\begin{aligned}
& f_{23}=\frac{\nu_{2} \Sigma_{2 f}}{\nu_{3} \Sigma_{3 f}} \phi_{23} \\
& f_{13}=\frac{\nu_{1} \Sigma_{1 f}}{\nu_{3} \Sigma_{3 f}} \phi_{13}
\end{aligned}
$$

Then we get:

$$
\begin{aligned}
\frac{\Sigma_{3}^{r e m}}{\chi_{3} \nu_{3} \Sigma_{3 f}} & =\frac{1}{k_{\infty}}\left[1+f_{23}+f_{13}\right] \\
\frac{1}{\chi_{2} \nu_{3} \Sigma_{3 f}}\left[\Sigma_{2}^{r e m} \phi_{23}-\Sigma_{23 s}\right] & =\frac{1}{k_{\infty}}\left[1+f_{23}+f_{13}\right] \\
\phi_{13} \Sigma_{1}^{r e m} & =\Sigma_{13 s}+\Sigma_{12 s} \phi_{23}
\end{aligned}
$$

If we substitute Eq. 56 into 57 and rearranging,

$$
\begin{aligned}
\frac{\Sigma_{2}^{r e m}}{\Sigma_{3}^{r e m}} \phi_{23} & =\frac{\Sigma_{23 s}}{\Sigma_{3}^{r e m}}+\frac{\chi_{2}}{\chi_{3}} \\
\frac{\Sigma_{1}^{r e m}}{\Sigma_{3}^{r e m}} \phi_{13} & =\frac{\Sigma_{13 s}}{\Sigma_{3}^{r e m}}+\frac{\Sigma_{12 s}}{\Sigma_{3}^{r m}} \phi_{23} \\
k_{\infty} & =\frac{\chi_{3} \nu_{3} \Sigma_{3 f}}{\Sigma_{3}^{r e m}}\left[1+f_{23}+f_{13}\right]
\end{aligned}
$$

Equations 57,58 , and 59 give the flux ratios and $k_{\infty}$. Tables 43,44 , and 45 give the cross sections used for the three-group problem. ${ }^{38}$

There are numerous cross sections involved in these equations, implying that there are numerous arbitrary choices we can make that will yield solutions to these equations. We show one set of cross sections that will satisfy a set of chosen conditions. ${ }^{38}$

If we make our basic choices as:

- $\mathrm{k}_{\infty}=1.600$

- $\chi_{3}=0.96, \chi_{2}=0.04, \chi_{1}=0.0$

- $5 \%$ of fission production occurs in group 3

- $20 \%$ of fission production occurs in group 2

- $75 \%$ of fission production occurs in group 1 
With these choices and the definitions of $f_{23}$ and $f_{13}$, we get:

$$
\begin{aligned}
& f_{23}=\frac{\nu_{2} \Sigma_{2 f}}{\nu_{3} \Sigma_{3 f}} \frac{\phi_{2}}{\phi_{3}}=4 \\
& f_{13}=\frac{\nu_{1} \Sigma_{1 f}}{\nu_{3} \Sigma_{3 f}} \frac{\phi_{1}}{\phi_{3}}=15
\end{aligned}
$$

Using this gives:

$$
\begin{aligned}
& \frac{\Sigma_{3}^{r e m}}{\nu_{3} \Sigma_{3 f}}=\frac{\chi_{3}}{k_{\infty}}\left[1+f_{23}+f_{13}\right] \\
& \frac{\Sigma_{3}^{r e m}}{\nu_{3} \Sigma_{3 f}}=12.0
\end{aligned}
$$

We can now make more arbitrary choices. If we choose:

- $\nu_{3}=3.0, \Sigma_{3 f}=0.006$

- $\nu_{2}=2.5, \Sigma_{2 f}=0.060$

- $\nu_{1}=2.0, \Sigma_{1 f}=0.900$

Then we get:

$$
\begin{aligned}
& \phi_{23}=0.480 \\
& \phi_{13}=0.150
\end{aligned}
$$

making $\Sigma_{3}^{r e m}=0.216$ from Eq. 63 . If we make more choices:

$$
\begin{aligned}
& \Sigma_{33 s}=0.024 \\
& \Sigma_{3 c}=0.006 \\
& \Sigma_{13 s}=0.033
\end{aligned}
$$

making $\Sigma_{3}=0.240$ and $\Sigma_{23 s}=0.171$. Using Eq. $57, \Sigma_{2}^{r e m}=0.375$. This result now gives: 


$$
\begin{aligned}
& \Sigma_{22 s}=0.600 \\
& \Sigma_{2 c}=0.040
\end{aligned}
$$

making $\Sigma_{2}=0.975$ and $\Sigma_{12 s}=0.275$. Using Eq. $58, \Sigma_{1}^{r e m}=1.10$. One last arbitrary choice is:

$$
\Sigma_{11 s}=2.00
$$

making $\Sigma_{1}=3.10$ and $\Sigma_{1 c}=0.20$.

\section{General Multigroup Infinite Medium $\mathbf{k}_{\infty}$}

More than three-group $\mathrm{k}_{\infty}$ derivations have been done (see Ref. 43). A general multigroup $\mathrm{k}_{\infty}$ derivation is included in this section for completeness. ${ }^{44}$

Given

$$
\overline{\overline{\Sigma_{t}}} \bar{\phi}=\overline{\overline{\Sigma_{s}}} \bar{\phi}+\frac{\bar{\chi} \overline{\nu \Sigma_{f}} \bar{\phi}}{k}
$$

where:

$$
\begin{aligned}
\overline{\overline{\Sigma_{t}}} & =G x G \text { matrix } \\
\overline{\overline{\Sigma_{s}}} & =G x G \text { matrix } \\
\bar{\chi} & =G x 1 \text { vector } \\
\overline{\nu \Sigma_{f}} & =1 x G \text { vector } \\
\bar{\phi} & =G x 1 \text { vector } \\
k & =\text { scalar }
\end{aligned}
$$

then:

$$
\begin{aligned}
\left(\overline{\overline{\Sigma_{t}}}-\overline{\overline{\Sigma_{s}}}\right) \bar{\phi} & =\frac{1}{k} \bar{\chi} \overline{\nu \Sigma_{f}} \bar{\phi} \\
\bar{\phi} & =\frac{1}{k}\left(\overline{\overline{\Sigma_{t}}}-\overline{\overline{\Sigma_{s}}}\right)^{-1} \bar{\chi} \overline{\nu \Sigma_{f}} \bar{\phi} \\
\overline{\nu \Sigma_{f}} \bar{\phi} & =\frac{1}{k} \overline{\nu \Sigma_{f}}\left(\overline{\overline{\Sigma_{t}}}-\overline{\overline{\Sigma_{s}}}\right)^{-1} \bar{\chi} \overline{\nu \overline{\Sigma_{f}}} \bar{\phi}
\end{aligned}
$$


Since $\nu \Sigma_{f} \phi$ is a scalar, it can be cancelled out and we get the followng explicit result:

$$
k=\overline{\nu \overline{\Sigma_{f}}}\left(\overline{\overline{\Sigma_{t}}}-\overline{\overline{\Sigma_{s}}}\right)^{-1} \bar{\phi}
$$

The right hand side of this equation is a scalar, equal to $\mathrm{k}$. Only one matrix inversion is necessary. 